\title{
Holographic gauge mediation via strongly coupled messengers
}

\author{
Paul McGuirk, ${ }^{1}$ Gary Shiu, ${ }^{1,2}$ and Yoske Sumitomo ${ }^{1,2}$ \\ ${ }^{1}$ Department of Physics, University of Wisconsin, Madison, WI 53706, USA \\ ${ }^{2}$ Institute for Advanced Study, Hong Kong University of Science and Technology, Hong \\ Kong, People's Republic of China \\ mcguirk@physics.wisc.edu, shiu@physics.wisc.edu, sumitomo@wisc.edu
}

\begin{abstract}
We consider a relative of semi-direct gauge mediation where the hidden sector exists at large 't Hooft coupling. Such scenarios can be difficult to describe using perturbative field theory methods but may fall into the class of holographic gauge mediation scenarios, meaning that they are amenable to the techniques of gauge/gravity duality. We use a recently found gravity solution to examine one such case where the hidden sector is a cascading gauge theory resulting in a confinement scale not much smaller than the messenger mass. In the original construction of holographic gauge mediation, as in other examples of semi-direct gauge mediation at strong coupling, the primary contributions to visible sector soft terms come from weakly coupled messenger mesons. In contrast to these examples, we describe the dual of a gauge theory where there are significant contributions from scales for which the strongly coupled messenger quarks are the effective degrees of freedom. In this regime, the visible sector gaugino mass can be calculated entirely from holography.
\end{abstract}

KEYWORDS: Gauge mediation, Gauge-gravity correspondence, D-branes. 


\section{Contents}

1. Introduction 1

2. Holographic gauge mediation 3

2.1 A non-SUSY deformation of Klebanov-Strassler

3. Gaugino masses from holography 13

3.1 Contributions from the 3-form flux

3.2 Contributions from the 5-form flux 24

3.3 Contributions from the perturbed spin connection 26

4. Conclusion 27

A. Conventions 30

B. Deformed conifold geometry 33

C. Pullbacks of bulk fields 37

\section{Introduction}

A major driving force behind the considerations of physics beyond the Standard Model (BSM) is arguably the hierarchy problem. Though countless number of scenarios have been proposed over the past few decades, they can be broadly divided depending on whether the unknown physics at the TeV scale is weakly or strongly coupled. Supersymmetry (SUSY) is a flagship example of the former. While the dynamics of a strongly coupled hidden sector is typically assumed to be the trigger of SUSY breaking, its influence on the Standard Model and its supersymmetric extension can be parametrized by a collection of operators that softly break SUSY. The perturbativity of such weakly coupled models not only makes them appealing in light of LEP constraints but also more amenable to quantitative studies. In comparison, strongly coupled scenarios such as technicolor involve strong coupling physics at the TeV scale and thus a detailed precision analysis of such models becomes a highly formidable task.

In supersymmetric scenarios one gains calculability by assuming that the BSM physics (i.e. the superpartners of the standard model or some extension) is weakly coupled but the large number of operators that must be added makes it difficult to make unique predictions (see e.g. [1, 2] for a review). The situation can be greatly ameliorated by studying the mechanism by which the effects of SUSY breaking are mediated to the visible sector. Of the 
different classes of mediation of SUSY breaking, gauge mediation [3]- [11] (see also [12] for a review and [13] for a very general discussion) has the advantage of suppressing the flavormixing effects that one would generically expect from the profusion of soft SUSY-breaking operators. In addition to the visible sector containing a supersymmetric extension of the standard model, such models possess fields that can be loosely divided into a hidden sector, which is neutral under the visible sector gauge group, and a messenger sector which is charged under the visible sector group. The hidden sector, either by design or by assumption obtains a SUSY-breaking state via strong dynamics (see e.g [14] for a review of dynamical SUSY breaking). The messenger sector, which couples to the hidden sector, communicates this effect to the visible sector fields via quantum effects.

Although in models of gauge mediation the messenger sector is often taken to be neutral with respect to hidden sector group responsible for the dynamical breaking of supersymmetry (in which case the coupling between the messengers and hidden sector typically occurs at the level of the superpotential), it is interesting to consider cases where this assumption is relaxed. In models of direct mediation, such as those in [15, 16, 17, 18], the distinction between the messengers and hidden sector is less sharp as the messengers are involved in the dynamical breaking of supersymmetry. Between these two extremes is semi-direct gauge mediation [19] in which the messengers are charged under the hidden sector gauge group, as well as the visible sector gauge group, but do not participate in the SUSY breaking. When the messenger sector is weakly coupled, one can use the language and techniques of perturbative field theory to calculate the effects on the visible sector. However, since SUSY breaking is often taken to occur via strong dynamics, one may wish to consider scenarios in which the hidden sector has a large 't Hooft coupling. In this case the messengers, when charged under the hidden sector group, are themselves strongly coupled and other techniques must be used. In recent years, our toolbox for handling strongly coupled gauge theories has expanded dramatically. Duality symmetries, such as Seiberg duality [20] and gauge/gravity duality [21, 22, 23], have enabled us to map strong coupling physics to their more tangible weak coupling duals. Armed with these tools, we can now explore new BSM scenarios and/or regions of model spaces which were previously overlooked or ignored because of the complications with strong coupling.

In this paper, we report on some progress in this direction by computing holographically the effects of semi-direct gauge mediated supersymmetry breaking with strongly coupled messengers. Though these models are weakly coupled in the sense that the effects of SUSY breaking on the visible sector can be expressed in terms of a soft SUSY-breaking Lagrangian, the fact that the messengers are strongly coupled with respect to the hidden sector gauge group suggests that their contributions to soft terms are subject to large hidden sector loop corrections. As a result, the way that the messenger mass and SUSY-breaking scale appear in the soft SUSY Langrangian may differ from the usual perturbative expressions (e.g. (2.5) and Fig. (1) which assume weakly coupled messengers. Fortunately, holographic techniques become useful when the hidden sector gauge group has large 't Hooft coupling'. In examples where the holographic dual is known, a tree-level computation on the gravity side amounts

\footnotetext{
${ }^{1}$ Holographic techniques may also be useful for describing strong coupling dynamics in the observable sector;
} 
to summing up all loop planar diagrams involving the messengers and the strongly coupled hidden sector (Fig. [3).

Our work is motivated by a related interesting scenario suggested in 25] and the gravity duals of SUSY-breaking large rank $\mathrm{SU}(N+M) \times \mathrm{SU}(N)$ gauge theories recently obtained in [26]. Utilizing the gravity background presented in [27], the authors of [25] constructed the holographic dual of a semi-direct gauge mediation scenario where the masses of the messenger quarks are much higher than the hidden sector confinement scale. The large residual $R$ symmetry preserved at high energies by the SUSY-breaking state suggests that contributions to the gaugino mass might be suppressed and indeed to leading order in the SUSY-breaking order parameter there is no contribution to the gaugino mass from physics at energies scales above the messenger quark mass in that scenario. Rather, the contributions to the gaugino mass come from below this scale where the effective degrees of freedom of the messenger sector are the weakly coupled mesonic bound states of the messenger quarks whose interactions are suppressed by the large 't Hooft coupling'. Therefore, even though the mesonic spectrum and effective $F$-terms require a holographic computation because the hidden sector is strongly coupled, the impact on the MSSM sector can be described by the usual perturbative expression. In contrast, in this paper we use the supergravity solutions obtained in [26] which allow us to work in a different kinematic regime where the messenger masses are comparable to the confinement scale. In this regime, we find a contribution from scales even above the messenger quark mass where the propagating degrees of freedom include strongly coupled quarks.

This paper is organized as follows. In Section 2, we review the holographic approach to gauge mediation suggested in [25]. Generic arguments based on $R$-symmetry motivate us to consider one of the non-supersymmetric solutions presented in 26] which we briefly summarize in Section 2.1. In Section 3, we compute the visible sector gaugino mass by considering gauginos living on a stack of probe D7-branes in this geometry. We end with some discussion in Section 1 . Some useful details about the deformed conifold geometry and our conventions are relegated to the appendices.

\section{Holographic gauge mediation}

As in 25], we take the hidden sector at short distances to be an $\mathcal{N}=1 \mathrm{SU}(N+M) \times \mathrm{SU}(N)$ gauge theory with large 't Hooft couplings. The matter content of the hidden sector possesses an $\mathrm{SU}(2) \times \mathrm{SU}(2)$ flavor symmetry under which the bifundamental chiral multiplets $A_{i=1,2}$ and $B_{i=1,2}$ transform as $(\mathbf{2}, \mathbf{1})$ and $(\mathbf{1}, \mathbf{2})$ respectively. The superpotential for this chiral matter is

$$
W_{\text {hidden }}=\lambda_{1} \epsilon^{i j} \epsilon^{k l} \operatorname{tr}\left(A_{i} B_{k} A_{j} B_{l}\right) \text {. }
$$

\footnotetext{
see, e.g. 24 for recent efforts toward describing a holographic and string theoretic embedding of technicolor.

${ }^{2}$ This is similar to [28] where composite states of analogous messenger fields were treated as the primary source of mediation.
} 
For non-vanishing $M$, as the theory flows to the IR, it undergoes a cascade of Seiberg dualities [20] ending with an $\mathrm{SU}(M)$ gauge theory exhibiting confinement at a scale $\Lambda_{\varepsilon}$. At short distances, the theory possesses a $\mathbb{Z}_{2 M} R$-symmetry that, in the IR, is spontaneously broken to $\mathbb{Z}_{2}$ by hidden sector gluino condensation.

At large 't Hooft coupling, the theory is strongly coupled and can be difficult to analyze. However, it is in this limit that the techniques of the gauge-gravity correspondence can be most reliably applied. The gravity dual for the high energy theory (the KT solution [29]) was constructed in IIB string theory ${ }^{3}$ by placing $N$ D3-branes and $M$ fractional D3-branes (i.e. D5-branes that wrap collapsing two-cycles) at a conifold singularity with the world-volumes filling the four external spacetime dimensions. The failure of the KT solution to describe the IR behavior of the field theory is related to the presence of the naked conifold singularity in the geometry. The KS solution [30] provides the IR resolution by smearing $M$ fractional D3-branes over the finite $S^{3}$ at the tip of the deformed conifold.

Although dynamical SUSY breaking in this theory is difficult to describe using standard field theory techniques, a holographic realization of a SUSY-breaking state can be constructed by adding $\overline{\mathrm{D} 3}$-branes to the tip. In the absence of D3-branes and as long as $P$, the number of $\overline{\mathrm{D} 3}$-branes, is much smaller than the amount of flux, the $\overline{\mathrm{D} 3}$-branes are perturbatively stable but will quantum mechanically tunnel into a SUSY-preserving vacuum 31]. The back reaction of the $\overline{\mathrm{D} 3}$-branes (the DKM solution) was found in [27] for the KT region (i.e. at large radius). The presence of the $\overline{\mathrm{D} 3}$-branes in the geometry explicitly breaks SUSY on the gravity side but the rapid fall-off of the resulting non-SUSY perturbations to the bulk fields due to the $\overline{\mathrm{D}}$-brane indicates that this configuration is dual to a metastable SUSY-breaking state, rather than dual to a theory that explicitly breaks SUSY.

The DKM solution was used in [25] to provide a holographic realization of a scenario of gauge mediation. The standard model gauge group is taken as a subgroup of a global $\mathrm{SU}(K)$ symmetry that is introduced into the field theory dual by adding a stack of $K$ probe D7-branes into the geometry and weakly gauged by adding a cutoff to the geometry (Fig. 1). These D7-branes fill the large four dimensional spacetime and extend along the radial direction of the conifold while wrapping a three-cycle in the angular directions. The matter content of the standard model is placed at the cutoff of the geometry, which on the gauge theory side corresponds to taking the standard model fields to be elementary fields, rather than composites resulting from the strong dynamics of the hidden sector.

In particular, the (deformed) conifold inherits a complex structure through the defining equation

$$
\sum_{i=1}^{4} z_{i}^{2}=\varepsilon^{2}, \quad z_{i} \in \mathbb{C},
$$

where the deformation parameter is related to the confining scale of the dual gauge theory by $\Lambda_{\varepsilon}=\varepsilon^{2 / 3}$. In terms of these holomorphic coordinates, we take the world-volume of the

\footnotetext{
${ }^{3}$ Our conventions are spelled out in Appendix A.
} 


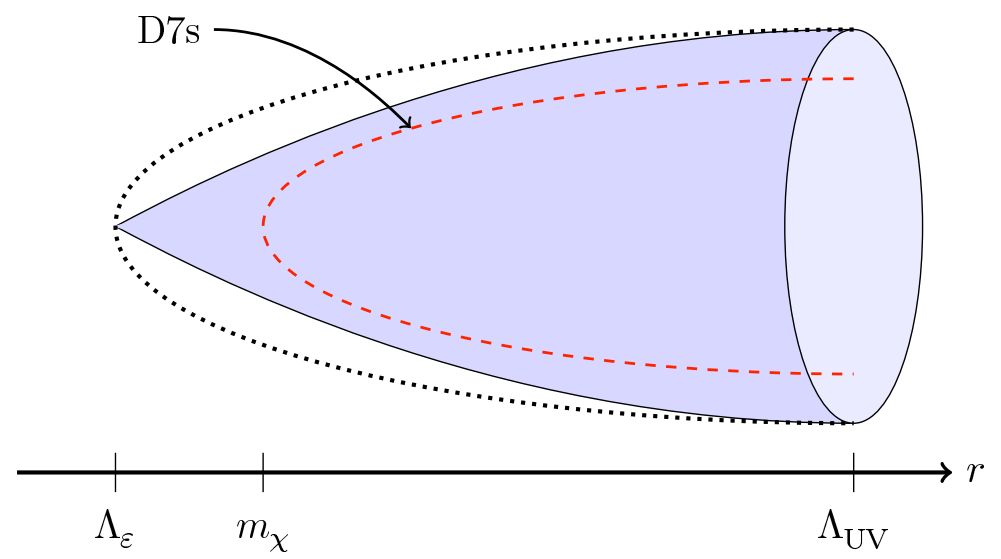

Figure 1: The flavor branes dip down to a distance set by the messenger mass $r=m_{\chi}$ where the coordinate $r$ is defined in (B.2) and has mass dimension 1. Although we will consider a case where $m_{\chi}$ is not much larger than $\Lambda_{\varepsilon}$, because of the strong warping this corresponds to a large proper distance. The back reaction of the $\overline{\mathrm{D} 3}$-branes reintroduces a singularity at $r=\Lambda_{\varepsilon}$ so that unlike the KS solution (represented by the dotted line), it is no longer smooth at the tip.

D7-branes to be specified by the condition

$$
z_{4}=\mu
$$

The addition of $K$ such D7-branes corresponds to the addition of the global SU $(K)$ flavor symmetry [32] and matter fields $\chi$ and $\tilde{\chi}$ to the gauge theory with $\chi$ transforming as an anti-fundamental of the $\mathrm{SU}(N)$ factor of the gauge theory and a fundamental of $\mathrm{SU}(K)$ and $\tilde{\chi}$ as the conjugate representations (Fig. 2). The bifundamental fields $A_{i}$ and $B_{i}$ couple to the quarks through the superpotential [33, 34]

$$
W_{\text {mess }}=\tilde{\chi}^{a}\left(A_{1} B_{1}+A_{2} B_{2}-\mu\right) \chi_{a}+\lambda_{2} \tilde{\chi} \chi \tilde{\chi} \chi .
$$

The fields $\chi$, which have mass dimension $\frac{3}{4}$, have mass $m_{\chi}=\mu^{2 / 3}$. This choice of embedding of the D7 world-volumes is made since although any holomorphically embedded D7 is supersymmetric in the (deformed) conifold, D7-branes satisfying a condition other than (2.3) typically require the existence of non-trivial world-volume flux to preserve the same supersymmetry as the KS solution [35].

Since $\chi$ and $\tilde{\chi}$ are charged under both the hidden sector and the visible sector, they are natural candidates for the messengers of the effect of SUSY breaking. Additionally, the SUSY-breaking state exists independently of the presence of the D7-branes implying that these messengers do not actively participate in the dynamical breaking of supersymmetry ${ }^{4}$. Thus, the setup is closely related to semi-direct gauge mediation [19], although $\chi$ and $\tilde{\chi}$ do have additional superpotential couplings to the hidden sector chiral matter. Because the

\footnotetext{
${ }^{4}$ This is at least true when the rank of the global symmetry group $K$ is much smaller than that of the gauge symmetry in which case the back reaction of the D7s on the geometry can be neglected.
} 


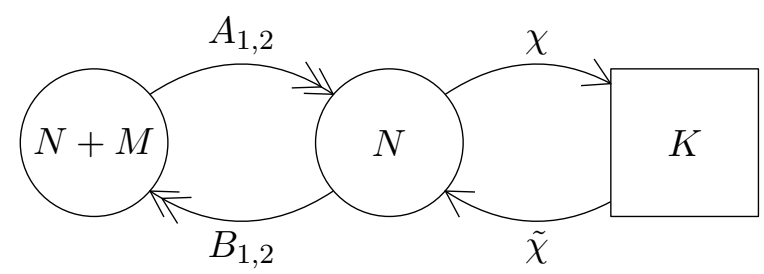

Figure 2: Quiver for the high energy theory. The standard model gauge group is a subgroup of the global SU $(K)$.

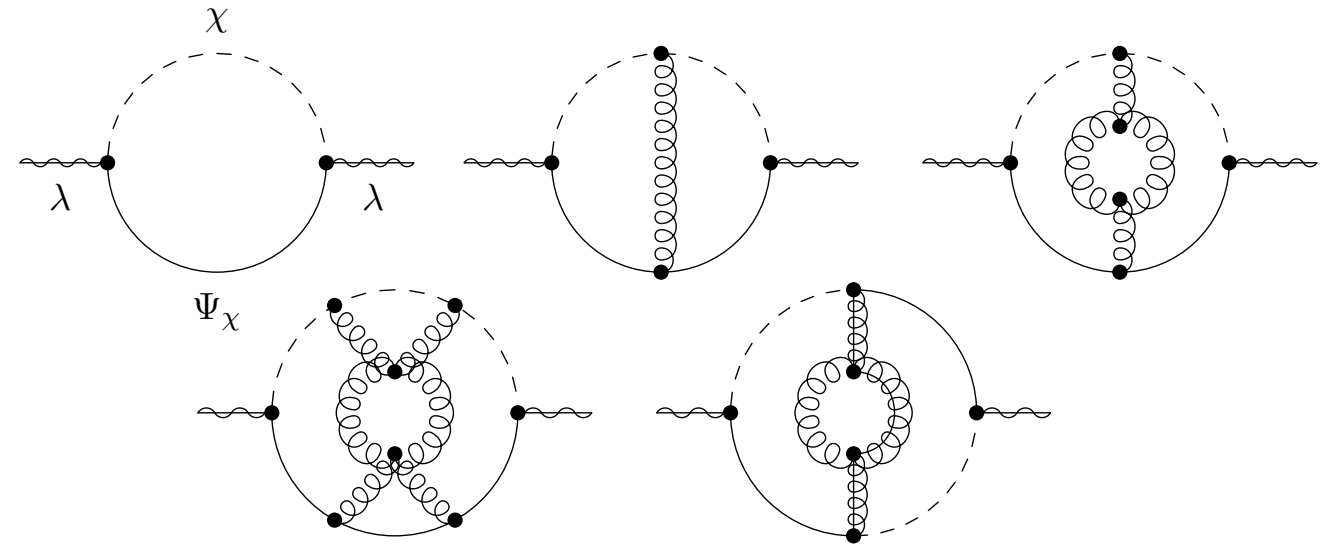

Figure 3: A very small sample of the infinite number of loops that might contribute to the visible sector gaugino mass. The gaugino couples to the messenger quarks $\Psi_{\chi}$ and squarks $\chi$ which also couple to the large 't Hooft coupling hidden sector gluons and gluinos. Since the hidden sector has large 't Hooft coupling, there are leading order contributions from planar diagrams with arbitrary numbers of loops. The calculation can be done holographically and, to leading order in the SUSYbreaking parameter, the loops cancel for $m_{\chi} \gg \Lambda_{\varepsilon}$. However, for $m_{\chi} \approx \Lambda_{\varepsilon}$, the cancellation no longer occurs.

messenger quarks are charged under the large 't Hooft hidden sector, there are potential contributions to the visible sector gaugino mass from all planar diagrams (Fig. 3).

Since the analysis of [25] was performed at large radius on the gravity side, the dual field theory is in a regime where the messengers are much heavier than the confining scale of the strongly coupled hidden sector, $m_{\chi} \gg \Lambda_{\varepsilon}$, and for many parts of that analysis, it is appropriate to neglect $\varepsilon$. In the absence of the deformation of the conifold singularity, the $R$-symmetry preserved by the geometry is $\mathbb{Z}_{2 M}$ [36, 37]. This large amount of $R$-symmetry suppresses contributions to the gaugino mass from scales above the messenger mass ${ }^{5}$. A non-vanishing messenger mass $\mu$, which has unit $R$-charge, breaks $R$-symmetry altogether. However, the $R$-symmetry breaking effect seems to be small and indeed in [25] the messenger

\footnotetext{
${ }^{5}$ The gaugino mass $m_{1 / 2}$ carries two units of $R$-charge, implying that any $R$-symmetry larger than $\mathbb{Z}_{2}$ forbids a non-vanishing $m_{1 / 2}$.
} 


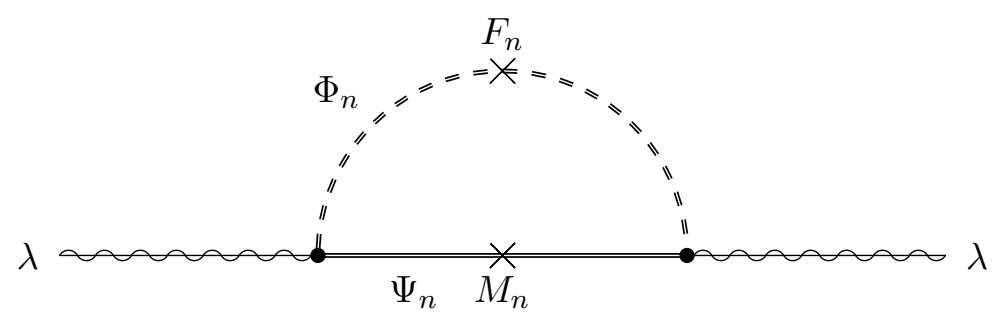

Figure 4: Contribution to the visible sector gaugino mass from a messenger mesons $\Phi_{n}$ and the superpartner mesinos $\Psi_{n}$. In the 't Hooft limit, the mesons and mesinos are weakly coupled and this diagram gives the leading order contribution from the mesons, giving (2.5).

quarks $\chi$ do not directly contribute to the gaugino mass until higher order in perturbation theory. As a result of the strong dynamics, the messenger $\chi$ fields bind into mesons $\Phi_{n}$ which are neutral under the hidden sector gauge group but transform as adjoints under the visible $\mathrm{SU}(K)$. The spectrum of mesons includes states whose masses are below $m_{\chi}$ and the SUSY-breaking dynamics of the hidden sector cause these meson superfields to feel $R$ symmetry breaking effective $F$-terms which lead to a non-vanishing gaugino mass. Because the hidden sector gauge group has large rank, the mesons are weakly coupled [38, 39, 40] and their physics can be described using standard field theory techniques, though the spectra $\left\{M_{n}, F_{n}\right\}$ of masses and $F$-terms do require a holographic calculation (which was performed via a DBI analysis in [25]). The mesons effectively act as messengers in a minimal gauge mediation scenario (Fig. (4) and the result of [25] is that the visible sector gauginos receive a mass

$$
m_{1 / 2}=\frac{g_{\mathrm{vis}}^{2} K}{16 \pi^{2}} \sum_{n} \frac{F_{n}}{M_{n}} \sim \frac{g_{\mathrm{vis}}^{2} K}{16 \pi^{2}} \frac{\Lambda_{\mathcal{S}}^{4}}{m_{\chi}^{3} \sqrt{4 \pi \lambda_{\text {eff }}\left(m_{\chi}\right)}} \sum_{n} n \mathrm{e}^{\mathrm{i} \theta_{n}},
$$

where $g_{\text {vis }}$ is the visible sector gauge coupling, $\Lambda_{\mathcal{S}}^{4}$ is the exponentially small vacuum energy of the SUSY-breaking state (which on the gravity side is set by the warped tension of the $\overline{\mathrm{D} 3}$-branes), $\lambda_{\text {eff }}\left(m_{\chi}\right)$ is the 't Hooft coupling of the hidden sector (dual to the amount of effective D3-charge) evaluated at the energy scale $m_{\chi}$, and $\theta_{n}$ are uncalculated phases. The summation is over a range of $n$ such that the effective field theory of weakly coupled mesons is appropriate. The effects of SUSY breaking is communicated to the remaining visible sector fields via gaugino mediation 41, 42].

\subsection{A non-SUSY deformation of Klebanov-Strassler}

In the far IR of the field theory, $R$-symmetry is broken down to $\mathbb{Z}_{2}$ by hidden sector gluino condensation. One would expect then that for $m_{\chi} \sim \Lambda_{\varepsilon}$, there will be contributions to the gaugino mass even from energies above $m_{\chi}$. Indeed, it was estimated in 25] that there should 
be a contribution to the gaugino mass from a finite deformation given by

$$
\delta m_{1 / 2} \sim \frac{\Lambda_{\varepsilon}}{m_{\chi}} \frac{\Lambda_{\mathcal{S}}^{4}}{m_{\chi}^{3} \sqrt{4 \pi \lambda_{\text {eff }}\left(m_{\chi}\right)}} .
$$

For $\mu \gg \varepsilon$ where the DKM solution is valid, this is much smaller than Eq. (2.5). In order for this contribution to be important compared to that of the meson messengers, it is necessary that $^{6}$

$$
\frac{\Lambda_{\varepsilon}}{m_{\chi}} \gtrsim \frac{g_{\mathrm{vis}}^{2} K}{16 \pi^{2}}
$$

As an estimate, we can suppose that the global symmetry has $K=5$ and forms an $\mathrm{SU}(5)$ GUT with $\alpha_{\mathrm{GUT}} \sim \frac{1}{25}$. This gives

$$
m_{\chi} \lesssim 60 \Lambda_{\varepsilon}
$$

Clearly, as the hierarchy between $\Lambda_{\varepsilon}$ and $m_{\chi}$ is reduced, the more important the $R$ breaking effects of confinement become. However, there is a possible concern with taking $m_{\chi}$ to be too small. On the gravity side of the calculation, decreasing $m_{\chi}$ corresponds to allowing the probe D7s to dip further into the throat, reaching smaller values of $\tau$. The presence of a $\overline{\mathrm{D} 3}$-brane introduces a curvature singularity into the back-reacted geometry at $\tau=0$ [26]. Such a singularity indicates the supergravity approximation of string theory breaks down and so the solution should be modified at distances below the string length. Thus, in order to trust our analysis of the gaugino mass, the D7-branes must not extend too deeply into the throat. For small radial distances, the KS metric (2.16) takes the approximate form ${ }^{7}$

$$
\mathrm{d} s_{10}^{2} \approx h_{0}^{-1 / 2} \eta_{\mu \nu} \mathrm{d} x^{\mu} \mathrm{d} x^{\nu}+h_{0}^{1 / 2}\left(\frac{1}{2} \mathrm{~d} \tau^{2}+\mathrm{d} \Omega_{3}^{2}+\frac{1}{4} \tau^{2}\left[g_{1}^{2}+g_{2}^{2}\right]\right),
$$

where $\mathrm{d} \Omega_{3}^{2}$ is the line element for a unit $S^{3}, g_{i}$ are other angular 1-forms, and $h_{0} \sim\left(g_{s} M\right)^{2}$.

We can estimate the string length for strings stretching along the radial direction at small $\tau$ by considering the world-sheet action,

$$
\begin{aligned}
S_{\sigma} & =-\frac{1}{2 \pi \alpha^{\prime}} \int_{\mathcal{M}} \mathrm{d}^{2} \sigma \sqrt{-\gamma} \gamma^{a b} g_{M N} \partial_{a} X^{M} \partial_{b} X^{N} \\
& \sim-\frac{g_{s} M}{2 \pi \alpha^{\prime}} \int_{\mathcal{M}} \mathrm{d}^{2} \sigma \sqrt{-\gamma} \gamma^{a b} \partial_{a} X^{\tau} \partial_{b} X^{\tau} .
\end{aligned}
$$

This implies that the effective string length for strings stretching along the holographic or internal angular directions is

$$
\sim \sqrt{\frac{2 \pi \alpha^{\prime}}{g_{s} M}}
$$

\footnotetext{
${ }^{6}$ Note that this only ensures that the contribution is comparable to that of a single meson, while many mesons contribute to (2.5).

${ }^{7}$ This metric does not quite satisfy the equations of motion; in addition to the existence of corrections that are higher order in $\tau$, there are also corrections that are $\mathcal{O}\left(\tau^{0}\right)$ but are negligible in the limit of large $g_{s} M$.
} 
The unknown stringy modification of the geometry can be neglected if the D7-branes remain much further than a string length from the location of the $\overline{\mathrm{D} 3}$-branes. Temporarily setting $2 \pi \alpha^{\prime}=1$, this condition becomes

$$
\tau_{\min } \gg \frac{1}{g_{s} M}
$$

In order for the supergravity approximation to be valid away from the $\operatorname{singularity,} g_{s} M$ must be large so the stringy resolution is important only for very small values of $\tau_{\min }$.

For a world-volume specified by the embedding condition (2.3), the D7-brane will extend to a minimum $\tau$ given by 34

$$
\tau_{\min }=2 \operatorname{arccosh} \frac{\mu}{\varepsilon}
$$

Combining (2.8) with (2.12) and using the relationships $\Lambda_{\varepsilon}=\varepsilon^{2 / 3}$ and $m_{\chi}=\mu^{2 / 3}$, we get the expectation that there will be important and calculable contributions to the gaugino mass when the D7-branes reach a minimum value $\tau_{\min }$ satisfying

$$
\frac{1}{g_{s} M} \ll \tau_{\min } \lesssim 14
$$

Given the relative complexity of the KS solution itself, an exact solution corresponding to the addition of an $\overline{\mathrm{D} 3}$-brane would be difficult to find. Instead, we will limit ourselves to a small $\tau$ expansion and take

$$
\frac{1}{g_{s} M} \ll \tau_{\min }<1
$$

In terms of the dual field theory variables, this means that we are taking the confining scale $\Lambda_{\varepsilon}$ and the messenger mass $m_{\chi}$ to be very near each other, but still requiring that latter be slightly larger. For simplicity, we take both $\varepsilon$ and $\mu$ to be real. The final result of our calculation will be a contribution to the gaugino mass that differs from (2.6) (which is not necessarily a contradiction since the result was obtained in a regime where $\Lambda_{\varepsilon} / m_{\chi}$ is a good expansion parameter while it is not for the calculation presented here). Nevertheless, (2.6) provides a good motivation to consider deformations to KS at small radius especially since our calculation will yield a contribution that is enhanced by the hidden sector 't Hooft coupling relative to the estimate 2.6 ).

In [26], we found small $\tau$ expansions for non-supersymmetric perturbations to the KS solution. For a choice of parameters, one of these solutions corresponds to the addition of $P$ D3- $\overline{\mathrm{D} 3}$ brane pairs smeared over the finite $S^{3}$ at the tip and is the small radius analogue of the DKM solution [27 $]^{8}$. In terms of the angular 1-forms $g_{i}$ (which are reviewed in Appendix B), the metric of this solution is of the warped type

$$
\mathrm{d} s_{10}^{2}=h^{-1 / 2}(\tau) \eta_{\mu \nu} \mathrm{d} x^{\mu} \mathrm{d} x^{\nu}+h^{1 / 2}(\tau) \mathrm{d} \tilde{s}_{6}^{2},
$$

where $\mu=0,1,2,3$ and where the radial and internal angular part of the metric is

$$
\mathrm{d} \tilde{s}_{6}^{2}=p(\tau) \mathrm{d} \tau^{2}+u(\tau) g_{5}^{2}+q(\tau)\left(g_{3}^{2}+g_{4}^{2}\right)+s(\tau)\left(g_{1}^{2}+g_{2}^{2}\right) .
$$

\footnotetext{
${ }^{8}$ The solution neglects in the supergravity limit the stringy annihilation of the pairs as was done in 27.
} 
Similar to what was found in [27], the presence of the $\overline{\mathrm{D} 3}$-branes "squashes" the unwarped $6 \mathrm{D}$ space so that it is no longer the geometry of the deformed conifold. Expanding the perturbation to leading order in $\tau$ gives

$$
\begin{array}{ll}
p(\tau)=p_{\mathrm{KS}}, & u(\tau)=u_{\mathrm{KS}}\left(1+\frac{u_{0}}{\tau}\right), \\
q(\tau)=q_{\mathrm{KS}}\left(1+\frac{q_{0}}{\tau}\right), & s(\tau)=s_{\mathrm{KS}}\left(1+\frac{s_{0}}{\tau}\right),
\end{array}
$$

where the Klebanov-Strassler solution has

$$
\begin{aligned}
& p_{\mathrm{KS}}(\tau)=u_{\mathrm{KS}}(\tau)=\frac{\varepsilon^{4 / 3}}{6 K^{2}(\tau)}, \quad q_{\mathrm{KS}}(\tau)=\frac{\varepsilon^{4 / 3}}{2} K(\tau) \cosh ^{2} \frac{\tau}{2}, \\
& s_{\mathrm{KS}}(\tau)=\frac{\varepsilon^{4 / 3}}{2} K(\tau) \sinh ^{2} \frac{\tau}{2}
\end{aligned}
$$

with

$$
K(\tau)=\frac{(\sinh 2 \tau-2 \tau)^{1 / 3}}{2^{1 / 3} \sinh \tau} .
$$

The presence of the $\overline{\mathrm{D} 3}$-branes perturbs the geometry so that

$$
u_{0} \sim q_{0} \sim s_{0} \sim \mathcal{S}
$$

where $\mathcal{S}$ is proportional to the number of $\mathrm{D} 3-\overline{\mathrm{D} 3}$ pairs

$$
\mathcal{S} \sim \frac{P \tau_{\mathrm{D} 3} \kappa_{10}^{2}}{\left(g_{s} M \alpha^{\prime}\right)^{2} \tilde{\mathcal{V}}_{\Omega}},
$$

where $\tau_{\mathrm{D} 3}$ is the tension of a D3 brane and $\tilde{\mathcal{V}}_{\Omega}$ is the unwarped volume of the $S^{3}$ at the tip ${ }^{9}$. Since our interest will be only in the parametric dependence of the gaugino mass, we will not need the more detailed expressions for the solution found in [26, Section 3.3].

The fractional D3-branes of the KS solution, together with the additional D3- $\overline{\mathrm{D} 3}$ pairs, produce non-trivial warping

$$
h(\tau)=h_{\mathrm{KS}}+\frac{h_{0}}{\tau}
$$

where

$$
h_{\mathrm{KS}}(\tau)=\left(g_{s} M \alpha^{\prime}\right)^{2} 2^{2 / 3} \varepsilon^{-8 / 3} I(\tau), \quad I(\tau)=\int_{\tau}^{\infty} \mathrm{d} x \frac{x \operatorname{coth} x-1}{\sinh ^{2} x}(\sinh 2 x-2 x)^{1 / 3},
$$

and

$$
h_{0} \sim\left(g_{s} M \alpha^{\prime}\right)^{2} \varepsilon^{-8 / 3} \mathcal{S} .
$$

The geometry exhibits a curvature singularity at $\tau=0$ where the Ricci scalar behaves as

$$
R \sim \frac{\mathcal{S}}{g_{s} M \tau}
$$

\footnotetext{
${ }^{9}$ This value parametrically differs from the analogous parameter in the DKM solution 27 by $\mathcal{S} \sim$ $\mathcal{S}^{\mathrm{DKM}} \varepsilon^{-8 / 3}$.
} 
The lower bound (2.12) also follows from demanding that the Ricci scalar remains small in string units.

The fluxes are

$$
\begin{aligned}
& B_{2}=\frac{g_{s} M \alpha^{\prime}}{2}\left[f(\tau) g_{1} \wedge g_{2}+k(\tau) g_{3} \wedge g_{4}\right], \\
& H_{3}=\frac{g_{s} M \alpha^{\prime}}{2}\left[\mathrm{~d} \tau \wedge\left(f^{\prime}(\tau) g_{1} \wedge g_{2}+k^{\prime}(\tau) g_{3} \wedge g_{4}\right)+\frac{1}{2}(k(\tau)-f(\tau)) g_{5} \wedge\left(g_{1} \wedge g_{3}+g_{2} \wedge g_{4}\right)\right] \\
& F_{3}=\frac{M \alpha^{\prime}}{2}\left[(1-F(\tau)) g_{5} \wedge g_{3} \wedge g_{4}+F(\tau) g_{5} \wedge g_{1} \wedge g_{2}+F^{\prime}(\tau) \mathrm{d} \tau \wedge\left(g_{1} \wedge g_{3}+g_{2} \wedge g_{4}\right)\right]
\end{aligned}
$$

with

$$
f(\tau)=f_{\mathrm{KS}}+f_{0}, \quad k(\tau)=k_{\mathrm{KS}}+\frac{k_{0}}{\tau^{2}}, \quad F(\tau)=F_{\mathrm{KS}}+\frac{F_{0}}{\tau},
$$

where the KS solution is

$$
\begin{aligned}
f_{\mathrm{KS}}(\tau) & =\frac{\tau \operatorname{coth} \tau-1}{2 \sinh \tau}(\cosh \tau-1), \quad k_{\mathrm{KS}}(\tau)=\frac{\tau \operatorname{coth} \tau-1}{2 \sinh \tau}(\cosh \tau+1), \\
F_{\mathrm{KS}}(\tau) & =\frac{\sinh \tau-\tau}{2 \sinh \tau}
\end{aligned}
$$

and again

$$
f_{0} \sim k_{0} \sim F_{0} \sim \mathcal{S}
$$

These source the R-R 5-form,

$$
F_{5}=\left(1+*_{10}\right) \mathcal{F}_{5}, \quad \mathcal{F}_{5}=\frac{g_{s} M^{2} \alpha^{\prime 2}}{4} \ell(\tau) g_{1} \wedge g_{2} \wedge g_{3} \wedge g_{4} \wedge g_{5}
$$

where

$$
\ell(\tau)=f(1-F)+k F .
$$

For the choice of parameters implicitly considered here, this solution, like that in [27], does not introduce a net amount of charge localized at the tip since the D3 and $\overline{\mathrm{D} 3}$-branes are added in pairs. However, $H_{3}$ and $F_{3}$ give rise to an effective D3 charge which is dual to the scale dependent effective 't Hooft coupling

$$
g_{s} N_{\text {eff }}(\tau) \sim\left(g_{s} M \alpha^{\prime}\right)^{2} \ell(\tau) .
$$

Finally, the SUSY-breaking 3-form fluxes give a non-trivial source for the dilaton,

$$
\Phi(\tau)=\log g_{s}+\Phi_{0} \tau
$$

where $\Phi_{0} \sim \mathcal{S}$. The axion in both KS and this perturbation is trivial, $C=0$.

The $\mathbb{Z}_{2} R$-symmetry is realized geometrically as a shift in an angle $\psi \rightarrow \psi+2 \pi$ (as briefly reviewed in Appendix B, $\psi$ ranges from 0 to $4 \pi$ ). Since the D3- $\overline{\mathrm{D} 3}$ pairs are smeared over 
the angular directions, the expressions for the bulk fields respect this shift symmetry. It is thus reasonable to assume that the SUSY-breaking state in the dual theory preserves the $\mathbb{Z}_{2}$ $R$-symmetry.

In [43, 44, 25], it was argued that the existence of a nonvanishing gaugino mass for the world-volume gauge theory living on a D7-brane is related to the existence of 3-form flux with Hodge type $(0,3)$. Using the relations between the 1-form $g_{i}$ and the holomorphic coordinates (2.2) reviewed in Appendix B, one can show that indeed the 3-form flux $G_{3}$ picks up such components in the above perturbation of KS. Using (B.9) and (B.11), the only non-vanishing component for $\mathrm{KS}$ is the $(2,1)$ component,

$$
\begin{aligned}
G_{3(\mathrm{KS})}^{(2,1)}=\frac{M \alpha^{\prime}}{2 \varepsilon^{6}} & {\left[\frac{\sinh 2 \tau-2 \tau}{\sinh ^{5} \tau}\left(\bar{z}_{m} \mathrm{~d} z_{m}\right) \wedge\left(\epsilon_{i j k \ell} z_{i} \bar{z}_{j} \mathrm{~d} z_{k} \wedge \mathrm{d} \bar{z}_{\ell}\right)\right.} \\
& \left.+\frac{2(1-\tau \operatorname{coth} \tau)}{\sinh ^{4} \tau}\left(z_{m} \mathrm{~d} \bar{z}_{m}\right) \wedge\left(\epsilon_{i j k \ell} z_{i} \bar{z}_{j} \mathrm{~d} z_{k} \wedge \mathrm{d} z_{\ell}\right)\right] .
\end{aligned}
$$

However, the above perturbation includes non-vanishing values for all components

$$
\begin{aligned}
& \delta G_{3}^{(2,1)} \sim \frac{\mathcal{S} M \alpha^{\prime}}{\varepsilon^{6} \tau^{5}}\left[c_{1}\left(\bar{z}_{m} \mathrm{~d} z_{m}\right) \wedge\left(\epsilon_{i j k \ell} z_{i} \bar{z}_{j} \mathrm{~d} z_{k} \wedge \mathrm{d} \bar{z}_{\ell}\right)+c_{2}\left(z_{m} \mathrm{~d} \bar{z}_{m}\right) \wedge\left(\epsilon_{i j k \ell} z_{i} \bar{z}_{j} \mathrm{~d} z_{k} \wedge \mathrm{d} z_{\ell}\right)\right] \\
& \delta G_{3}^{(1,2)} \sim \frac{\mathcal{S} M \alpha^{\prime}}{\varepsilon^{6} \tau^{5}}\left[c_{3}\left(z_{m} \mathrm{~d} \bar{z}_{m}\right) \wedge\left(\epsilon_{i j k \ell} z_{i} \bar{z}_{j} \mathrm{~d} z_{k} \wedge \mathrm{d} \bar{z}_{\ell}\right)+c_{4}\left(\bar{z}_{m} \mathrm{~d} z_{m}\right) \wedge\left(\epsilon_{i j k \ell} z_{i} \bar{z}_{j} \mathrm{~d} \bar{z}_{k} \wedge \mathrm{d} \bar{z}_{\ell}\right)\right] \\
& \delta G_{3}^{(3,0)} \sim \frac{c_{5} \mathcal{S} M \alpha^{\prime}}{\varepsilon^{6} \tau^{3}}\left(\bar{z}_{m} \mathrm{~d} z_{m}\right) \wedge\left(\epsilon_{i j k \ell} z_{i} \bar{z}_{j} \mathrm{~d} z_{k} \wedge \mathrm{d} z_{\ell}\right) \\
& \delta G_{3}^{(0,3)} \sim \frac{c_{6} \mathcal{S} M \alpha^{\prime}}{\varepsilon^{6} \tau^{3}}\left(z_{m} \mathrm{~d} \bar{z}_{m}\right) \wedge\left(\epsilon_{i j k \ell} z_{i} \bar{z}_{j} \mathrm{~d} \bar{z}_{k} \wedge \mathrm{d} \bar{z}_{\ell}\right) .
\end{aligned}
$$

where the $c_{i}$ are non-vanishing $\mathcal{O}(1)$ coefficients whose exact values we will not need. In contrast, only $(1,2)$ and $(2,1)$ components appeared in the large radius solution of [27].

It was shown in [25] that if the complex structure of the space changes, then the existence of $g_{z z}$ and $g_{\bar{z} \bar{z}}$ components of the metric can give rise to additional contributions to the gaugino mass. Such components exist in this perturbation. Using (B.12), the unwarped metric for the holographic and internal radial directions for the KS metric is Calabi-Yau

$$
\mathrm{d} \tilde{s}_{6}^{2}=\left(\partial_{i} \partial_{\bar{j}} \mathcal{F}\right) \mathrm{d} z_{i} \mathrm{~d} \bar{z}_{j}, \quad \mathcal{F}^{\prime}\left(\varepsilon^{2} \cosh \tau\right)=\varepsilon^{-2 / 3} K(\tau),
$$

while the perturbation to the metric is not even Hermitian with respect to the original complex structure

$$
\begin{aligned}
\left(\varepsilon^{4} \sinh ^{2} \tau\right) \delta\left(\mathrm{d} \tilde{s}_{6}^{2}\right) \sim & \frac{\mathcal{S} \varepsilon^{4 / 3}}{\tau}\left[d_{1}\left(\left(\bar{z}_{i} \mathrm{~d} z_{i}\right)^{2}+\left(z_{i} \mathrm{~d} \bar{z}_{i}\right)^{2}\right)+d_{2}\left(\bar{z}_{i} \mathrm{~d} z_{i}\right)\left(z_{i} \mathrm{~d} \bar{z}_{i}\right)\right] \\
& +\mathcal{S} \varepsilon^{10 / 3} \tau\left[d_{3}\left(\mathrm{~d} z_{i} \mathrm{~d} z_{i}+\mathrm{d} \bar{z}_{i} \mathrm{~d} \bar{z}_{i}\right)+d_{4} \mathrm{~d} z_{i} \mathrm{~d} \bar{z}_{i}\right]
\end{aligned}
$$

where the $d_{i}$ are another set of $\mathcal{O}(1)$ coefficients. 
For the purposes of calculating the gaugino mass, it is useful to introduce another set of holomorphic 1-forms $\mathrm{d} Z_{i}(\mathrm{~B} .14)$. Using (B.17) and (B.18), the components of $G_{3}$ can be written in these coordinates as

$$
\begin{aligned}
G_{3(\mathrm{KS})}=-\frac{M \alpha^{\prime}}{16 \sinh ^{2} \tau}[ & 4(\sinh \tau-\tau \cosh \tau) \mathrm{d} \bar{Z}_{1} \wedge \mathrm{d} Z_{2} \wedge \mathrm{d} Z_{3} \\
& \left.+(\sinh 2 \tau-2 \tau)\left(\mathrm{d} Z_{1} \wedge \mathrm{d} Z_{2} \wedge \mathrm{d} \bar{Z}_{3}-\mathrm{d} Z_{1} \wedge \mathrm{d} \bar{Z}_{2} \wedge \mathrm{d} Z_{3}\right)\right]
\end{aligned}
$$

while the perturbation to $G_{3}$ has components

$$
\begin{aligned}
& \delta G_{3}^{(2,1)} \sim-\frac{\mathcal{S} M \alpha^{\prime}}{4 \tau^{2}}\left[c_{1}\left(\mathrm{~d} Z_{1} \wedge \mathrm{d} Z_{2} \wedge \mathrm{d} \bar{Z}_{3}-\mathrm{d} Z_{1} \wedge \mathrm{d} \bar{Z}_{2} \wedge \mathrm{d} Z_{3}\right)+c_{2} \mathrm{~d} \bar{Z}_{1} \wedge \mathrm{d} Z_{2} \wedge \mathrm{d} Z_{3}\right] \\
& \delta G_{3}^{(1,2)} \sim+\frac{\mathcal{S} M \alpha^{\prime}}{4 \tau^{2}}\left[c_{3}\left(\mathrm{~d} \bar{Z}_{1} \wedge \mathrm{d} \bar{Z}_{2} \wedge \mathrm{d} Z_{3}-\mathrm{d} \bar{Z}_{1} \wedge \mathrm{d} Z_{2} \wedge \mathrm{d} \bar{Z}_{3}\right)+c_{4} \mathrm{~d} Z_{1} \wedge \mathrm{d} \bar{Z}_{2} \wedge \mathrm{d} \bar{Z}_{3}\right] \\
& \delta G_{3}^{(3,0)} \sim-\left(c_{5} \mathcal{S} M \alpha^{\prime}\right) \mathrm{d} Z_{1} \wedge \mathrm{d} Z_{2} \wedge \mathrm{d} Z_{3} \\
& \delta G_{3}^{(0,3)} \sim+\left(c_{6} \mathcal{S} M \alpha^{\prime}\right) \mathrm{d} \bar{Z}_{1} \wedge \mathrm{d} \bar{Z}_{2} \wedge \mathrm{d} \bar{Z}_{3} .
\end{aligned}
$$

The metric in these coordinates is $(\mathrm{B} .15)$. In $\mathrm{KS}$, this becomes

$$
\mathrm{d} \tilde{s}_{6}^{2}=\frac{\varepsilon^{4 / 3}}{6 K^{2}} \mathrm{~d} Z_{1} \mathrm{~d} \bar{Z}_{2}+\frac{\varepsilon^{4 / 3} K}{2} \sinh ^{2} \frac{\tau}{2} \mathrm{~d} Z_{2} \mathrm{~d} \bar{Z}_{2}+\frac{\varepsilon^{4 / 3} K}{2} \cosh ^{2} \frac{\tau}{2} \mathrm{~d} Z_{3} \mathrm{~d} \bar{Z}_{3},
$$

while the perturbation to the metric is

$$
\begin{aligned}
\delta\left(\mathrm{d} \tilde{s}_{6}^{2}\right) \sim & \mathcal{S} \varepsilon^{4 / 3}\left[\frac{\hat{d}_{1}}{\tau} \mathrm{d} Z_{1} \mathrm{~d} \bar{Z}_{1}+\hat{d}_{2} \tau \mathrm{d} Z_{2} \mathrm{~d} \bar{Z}_{2}+\frac{\hat{d}_{3}}{\tau} \mathrm{d} Z_{3} \mathrm{~d} \bar{Z}_{3}\right. \\
& \left.+\frac{\hat{d}_{4}}{\tau}\left(\mathrm{d} Z_{1} \mathrm{~d} Z_{1}+\mathrm{d} \bar{Z}_{1} \mathrm{~d} \bar{Z}_{1}\right)+\hat{d}_{5} \tau\left(\mathrm{d} Z_{2} \mathrm{~d} Z_{2}+\mathrm{d} \bar{Z}_{2} \mathrm{~d} \bar{Z}_{2}\right)+\frac{\hat{d}_{6}}{\tau}\left(\mathrm{d} Z_{3} \mathrm{~d} Z_{3}+\mathrm{d} \bar{Z}_{3} \mathrm{~d} \bar{Z}_{3}\right)\right]
\end{aligned}
$$

where the $\hat{d}_{i}$ are $\mathcal{O}(1)$ coefficients that can be written in terms of $d_{i}$.

\section{Gaugino masses from holography}

Using the above SUSY-breaking gravity solution, we can now proceed to calculate the mass of a gaugino living on a stack of $K$ probe D7-branes in this geometry. In order to neglect the back reaction of the D7-branes, we take $K \ll P \ll M$. Although it would be interesting to calculate the back reaction as in [33, 45, 46], such a calculation would lead to a self-energy problem when we try to calculate the mass of a gaugino living on the D7s. The calculation here is similar that of [25] though because of the reduced isometry of the geometry (which in the dual field theory corresponds to reduced $R$-symmetry in the hidden sector) it leads to a non-vanishing result even to leading order in $\mathcal{S}$.

The starting point is the Dirac-like action for a D7-brane presented in [47] based largely

on [48, 49] and reviewed in Appendix A. This action is strictly speaking only valid in the 
Abelian (i.e. $K=1$ ) case, but in the supergravity limit, we do not expect any deviations for the gaugino mass from the Abelian result ${ }^{10}$. The strategy is to find the effective mass for the gaugino that results from a dimensional reduction of the world-volume action to $\mathbb{R}^{1,3}$, which in the dual field theory corresponds to calculating the mass resulting from all planar diagrams in the 't Hooft limit. We begin with an analysis of contributions to the gaugino mass from 3 -form flux (some of which are non-vanishing). Similar considerations were performed in 43] and [25]. However, a priori there could be additional contributions from other bulk fields which we consider towards the end of this section.

In the KS background, the 3 -form flux is $\operatorname{ISD}(2,1)$ and the gaugino remains massless 43 ]. Thus contributions to the gaugino mass will come from the non-SUSY perturbations to KS. Since the solution is known only to leading order in $\mathcal{S}$, we will be interested only in contributions to the gaugino mass that are also linear in $\mathcal{S}$.

\subsection{Contributions from the 3 -form flux}

The fermionic action is written in terms of a bispinor

$$
\Theta=\left(\begin{array}{c}
\theta \\
\tilde{\theta}
\end{array}\right),
$$

where $\theta$ and $\tilde{\theta}$ are 10D Majorana-Weyl spinors of positive chirality. For the probe D7-branes, the contribution to the Dirac action from the 3-form flux can be written as a trace over gauge indices

$$
\begin{aligned}
S_{\mathrm{D} 7}^{(3)}=\frac{\mathrm{i} \tau_{\mathrm{D} 7} g_{s}^{-1 / 2}}{8} & \int \mathrm{d}^{8} \xi \mathrm{e}^{3 \Phi / 2} \sqrt{|\operatorname{det} \mathcal{M}|} \\
& \times \operatorname{tr}\left\{\bar{\Theta} P_{-}^{\mathrm{D} 7}\left[2 \mathcal{G}_{3}^{+}+\left(\hat{\mathcal{M}}^{-1}\right)^{\alpha \beta} \Gamma_{\beta}\left(\mathcal{G}_{3}^{-} \Gamma_{\alpha}+\frac{1}{2} \Gamma_{\alpha}\left(\mathcal{G}_{3}^{-}-\mathcal{G}_{3}^{+}\right)\right)\right] \Theta\right\},
\end{aligned}
$$

where in the absence of world-volume flux,

$$
\mathcal{M}_{\alpha \beta}=\gamma_{\alpha \beta}+g_{s}^{1 / 2} \mathrm{e}^{-\Phi / 2} b_{\alpha \beta}
$$

with $\gamma$ and $b$ the pullbacks of the metric and the NS-NS 2-form and

$$
\hat{\mathcal{M}}_{\alpha \beta}=\left(\begin{array}{cc}
\mathcal{M}_{\beta \alpha} & 0 \\
0 & \mathcal{M}_{\alpha \beta}
\end{array}\right),
$$

where $\xi^{\alpha}$ are the world-volume coordinates and tensors with indices $\alpha, \beta$ denote pullbacks onto the world-volumes of the branes. $x^{\mu}$ denotes a coordinate in the four large spacetime

\footnotetext{
${ }^{10}$ The fermionic $\mathrm{D} p$-brane action should also contain a Yukawa-like coupling $\operatorname{tr}\{\lambda,[\Phi, \lambda]\}$ where the $\Phi$ are the transverse fluctuations of D7-branes. However, such a term only contributes to the gaugino mass at loop level on the gravity side which corresponds to a finite 't Hooft coupling effect on the field theory side. Indeed, this term gives a coupling between the gaugino and the meson messengers $\Phi_{n}$ and as shown in 25] gives a contribution suppressed by the 't Hooft coupling.
} 
dimensions while $x^{a}$ are coordinates on the radial direction and the internal angular directions. When acting on the gaugino in the supersymmetric case, the projection operator can be written as $P_{ \pm}^{\mathrm{D} 7}=\frac{1}{2}\left(1 \mp \Gamma_{\mathrm{D} 7}\right)$ with 47,50

$$
\Gamma_{\mathrm{D} 7}=\left(\begin{array}{cc}
0 & \mathrm{i} \Gamma_{(8)} \\
-\mathrm{i} \Gamma_{(8)} & 0
\end{array}\right),
$$

where $\Gamma_{(8)}$ is the usual 8D chirality operator. The solution presented in Section 2.1 is no longer BPS, but the deviation from Eq. (3.5) essentially gives a mixing term and so contributes to the gaugino mass at higher order in $\mathcal{S}$. Finally the contribution from the 3 -form flux is

$$
\mathcal{G}_{3}^{ \pm}=\frac{1}{3 !}\left(\tilde{F}_{M N P} \sigma_{1} \pm \mathrm{e}^{-\Phi} H_{M N P} \sigma_{3}\right) \Gamma^{M N P} .
$$

As is well known, the fermionic part of the action has a redundant description of the fermionic degrees of freedom known as $\kappa$-symmetry. We choose to eliminate the redundancy by taking the particular $\kappa$-fixing condition $\tilde{\theta}=0$

$$
\Theta=\left(\begin{array}{l}
\theta \\
0
\end{array}\right) .
$$

To leading order in $\mathcal{S}$, we can take the gaugino wavefunction to be unperturbed by the addition of the $\overline{\mathrm{D} 3}$-branes in which case it is given by [50]

$$
\theta\left(x^{\alpha}\right)=\lambda\left(x^{\mu}\right) \otimes h^{3 / 8} \eta\left(x^{a}\right),
$$

where $\eta$ is covariantly constant with respect to the underlying Calabi-Yau (i.e. deformed conifold) metric and is annihilated by the holomorphic $\Gamma$-matrices $\Gamma_{z}$ [51]. Taking $\theta$ to have negative $6 \mathrm{D}$ chirality, the $4 \mathrm{D}$ chirality is also negative

$$
\Gamma_{(4)} \theta=\mathrm{i} \Gamma^{0123} \theta=-\theta \text {. }
$$

As shown in [50], the gaugino has positive chirality with respect to the chirality operator for the internal 4-cycle wrapped by the D7-brane, $\Gamma^{\text {extra }}$. This gives a positive $8 \mathrm{D}$ chirality.

$$
\Gamma_{(8)} \theta=-\Gamma_{(4)} \Gamma^{\mathrm{extra}} \theta=\theta \text {. }
$$

Using the above choice of $\kappa$-fixing, we find that the action (3.2) is

$$
\begin{aligned}
S_{\mathrm{D} 7}^{(3)}=- & \frac{\tau_{\mathrm{D} 7} g_{s}^{-1 / 2}}{8 \cdot 3 !} \int \mathrm{d}^{8} \xi \mathrm{e}^{3 \Phi / 2} \sqrt{|\operatorname{det} \mathcal{M}|} \operatorname{tr}\left\{\overline { \theta } \left[G_{M N P} \Gamma^{M N P}\right.\right. \\
& \left.+\left(\mathcal{M}^{-1}\right)^{(\alpha \beta)} \Gamma_{\beta}\left(G_{M N P}^{*} \Gamma^{M N P} \Gamma_{\alpha}+\frac{1}{2} \Gamma_{\alpha}\left(G_{M N P}^{*}-G_{M N P}\right) \Gamma^{M N P}\right)\right] \\
& \left.\left.+\left(\mathcal{M}^{-1}\right)^{[\alpha \beta]} \Gamma_{\beta}\left(G_{M N P} \Gamma^{M N P} \Gamma_{\alpha}+\frac{1}{2} \Gamma_{\alpha}\left(G_{M N P}-G_{M N P}^{*}\right) \Gamma^{M N P}\right)\right] \theta\right\}
\end{aligned}
$$


with

$$
G_{3}=\tilde{F}_{3}-\mathrm{ie}^{-\Phi} H_{3}, \quad G_{3}^{*}=\tilde{F}+\mathrm{ie}^{-\Phi} H_{3},
$$

and $(\alpha \beta)$ and $[\alpha \beta]$ indicate symmetrization and anti-symmetrization over the indices.

To find contributions to the gaugino mass, we must consider perturbations to the fields in (3.11). The perturbations to consider are those of the measure $\mathrm{e}^{3 \Phi / 2} \sqrt{|\operatorname{det} \mathcal{M}|}$, the metric $g_{M N}, \mathcal{M}_{\alpha \beta}$, and the 3 -form flux $G_{3}$ (as discussed below, perturbations of the $\Gamma$-matrices contribute only higher order terms to the gaugino mass). Moreover to leading order in $\mathcal{S}$, we need only to consider the perturbations to one of these at a time.

\subsubsection{Contributions from the perturbed 3-form flux}

We first consider the contributions from the perturbed flux but unperturbed metric and in particular consider the term

$$
\operatorname{tr}\left\{\bar{\theta} G_{M N P} \Gamma^{M N P} \theta\right\}
$$

$G_{3}$ has legs only on the holographic and internal directions so

$$
\operatorname{tr}\left\{\bar{\theta} G_{M N P} \Gamma^{M N P} \theta\right\}=\operatorname{tr}\left(\lambda^{2}\right) \tilde{g}^{m n} \tilde{g}^{s r} \tilde{g}^{p q} G_{m s p} \eta^{T} \tilde{\Gamma}_{n r q} \eta
$$

where we have used (3.8) and related the warped $\Gamma$-matrices to the unwarped ones

$$
\Gamma_{m}=h^{1 / 4} \tilde{\Gamma}_{m},
$$

and $\tilde{g}$ is the unwarped bulk metric. Since $\tilde{\Gamma}_{z} \eta=0$, this becomes

$$
\operatorname{tr}\left\{\bar{\theta} G_{M N P} \Gamma^{M N P} \theta\right\}=\operatorname{tr}\left(\lambda^{2}\right) \tilde{g}^{\bar{i}^{\prime}} \tilde{g}^{j \bar{j}^{\prime}} \tilde{g}^{k \bar{k}^{\prime}} G_{i j k} \eta^{T} \tilde{\Gamma}_{\bar{i}^{\prime} \bar{j}^{\prime} \bar{k}^{\prime}} \eta
$$

where $i, j, k$ are holomorphic indices and $\bar{i}, \bar{j}, \bar{k}$ are anti-holomorphic. Terms that involve $\Gamma$ matrices of mixed types (e.g. $\Gamma_{\bar{i}} \Gamma_{j} \Gamma_{\bar{k}}$ ) give rise to mixing terms and so contribute to the gaugino mass at higher order in the perturbation. Eq. (3.16) implies that in addition to the $(0,3)$ contribution to the gaugino mass argued to exist in [43] and coming from (3.35), there is a contribution from the $(3,0)$-component. $\eta$ is covariantly constant with respect to the Calabi-Yau metric which allows us to write ${ }^{11}$

$$
\eta^{T} \tilde{\Gamma}_{\bar{i} \bar{j} \bar{k}} \eta=\bar{\Omega}_{\bar{i} \bar{j} \bar{k}}
$$

where $\Omega$ is the holomorphic 3 -form of the underlying Calabi-Yau. Thus, there is a contribution to the gaugino mass of the form

$$
-\frac{\tau_{\mathrm{D} 7} g_{s}^{-1 / 2}}{8 \cdot 3 !} \int_{\mathrm{R}^{1,3}} \mathrm{~d}^{4} x \operatorname{tr}\left(\lambda^{2}\right) \int_{\Sigma_{4}} \mathrm{~d}^{4} x \mathrm{e}^{3 \Phi / 2} \sqrt{|\operatorname{det} \mathcal{M}|} \bar{\Omega}^{\widetilde{i j k}} G_{i j k} .
$$

\footnotetext{
${ }^{11}$ We note that here it is especially important that the derivative appearing in the Dirac-like action is the pullback of the covariant derivative built from the bulk metric and not the covariant derivative built from the pullback of the metric.
} 
where $\tilde{i}$, etc denote indices raised with the unwarped metric $\tilde{\gamma}$ and $\Sigma_{4}$ denotes the 4-cycle wrapped by the D7.

Since we have only calculated the perturbations due to the $\overline{\mathrm{D} 3}$-branes as a small $\tau$ expansion, we cannot calculate the gaugino mass exactly and will therefore only be interested in a parametric dependence. Using (2.34d) and (B.13), we find

$$
\frac{1}{3 !} \bar{\Omega}^{\widetilde{i j k}} G_{i j k} \sim \frac{M \alpha^{\prime}}{\varepsilon^{2} \tau} \mathcal{S}+\mathcal{O}(\tau)
$$

The terms that are higher order in $\tau$ have been omitted since the integral in (3.18) will receive contributions only for small $\tau$.

Because we are expanding to linear order in $\mathcal{S}$, all other fields are set to their background (KS) values. Expressions for the pullbacks of the metric and NS-NS 2-form are given in Appendix C. Even though it is possible to write an exact expression for $\operatorname{det} \mathcal{M}$, it is relatively complex and because we are interested only in the parametric dependence, we will consider only the behavior for small $\tau$. To illustrate the approximation we use for $\operatorname{det} \mathcal{M}$ and other fields, we first consider the determinant of the induced metric $\gamma$ which has a simpler exact expression. In 25] it was shown that the determinant of the pulled-back metric is

$$
\gamma=\frac{K^{4}\left(\mu^{2}-\varepsilon^{2}\right)^{4}}{16 \varepsilon^{8 / 3}} K_{2}^{2} \cosh ^{2} \frac{\rho}{2} \sinh ^{2} \frac{\rho}{2}
$$

where

$$
\begin{aligned}
\varepsilon^{2} \cosh \tau & =\left(\mu^{2}-\varepsilon^{2}\right) \cosh \rho+\mu^{2}, \\
K(\tau) & =\frac{(\sinh 2 \tau-2 \tau)^{1 / 3}}{2^{1 / 3} \sinh \tau}, \\
K_{2}(\tau) & =\cosh \rho-\frac{\left(\mu^{2}-\varepsilon^{2}\right) \sinh ^{2} \rho}{\varepsilon^{2} \sinh ^{2} \tau}\left(\cosh \tau-\frac{2}{3 K^{3}}\right) .
\end{aligned}
$$

The stack of D7s extends to a minimum value of $\tau$ given by

$$
\tau_{\min }=2 \operatorname{arccosh} \frac{\mu}{\varepsilon}
$$

and the integral (3.18) should be dominated by contributions from $\tau$ near this value since the SUSY-breaking fluxes are peaked at small $\tau$. Expanding $(3.20)$ about $\tau=\tau_{\min }$, we find

$$
\gamma=\frac{1}{64 \cdot 2^{1 / 3} \varepsilon^{2 / 3}} \frac{\left(\mu^{2}-\varepsilon^{2} \cosh \tau_{\min }\right)^{3}}{\sinh ^{3} \tau_{\min }}\left(\sinh 2 \tau_{\min }-2 \tau_{\min }\right)^{4 / 3}\left(\tau-\tau_{\min }\right)+\mathcal{O}\left(\left(\tau-\tau_{\min }\right)^{2}\right) .
$$

To trust that the small $\tau$ expansion is good, we must have that $\tau_{\min }<1$ which implies that $\mu$ cannot be much larger than $\varepsilon$. For small $\tau_{\min }$,

$$
\mu \approx \varepsilon\left(1+\frac{1}{8} \tau_{\min }^{2}\right)
$$


Then $\gamma$ takes the approximate form

$$
\gamma \approx \frac{2^{5 / 6}}{3^{4 / 3}} \varepsilon^{16 / 3} \tau_{\min }^{7}\left(\tau-\tau_{\min }\right)
$$

where higher order terms in $\tau_{\text {min }}$ have been dropped. Following a similar process for $\mathcal{M}$ gives the same parametric dependence

$$
\sqrt{|\operatorname{det} \mathcal{M}|} \sim \varepsilon^{8 / 3} \tau_{\min }^{7 / 2}\left(\tau-\tau_{\min }\right)^{1 / 2}
$$

Combining this with (3.19), (3.18) becomes

$$
\sim-\tau_{\mathrm{D} 7} g_{s} \int_{\mathbb{R}^{1,3}} \mathrm{~d}^{4} x \operatorname{tr}\left(\lambda^{2}\right) \int_{\tau_{\min }}^{\tau_{\max }} \mathrm{d} \tau \varepsilon^{8 / 3} \tau_{\min }^{7 / 2}\left(\tau-\tau_{\min }\right)^{1 / 2} \frac{M \alpha^{\prime}}{\varepsilon^{2} \tau} \mathcal{S}
$$

where we have omitted the angular integrals since they do not contribute to the parametric dependence and where $\tau_{\max }$ represents some UV cutoff for the field theory. Defining $t=$ $\tau / \tau_{\min }$, we find

$$
\sim-\tau_{\mathrm{D} 7} g_{s} M \alpha^{\prime} \varepsilon^{2 / 3} \tau_{\min }^{4} \mathcal{S} \int_{\mathbb{R}^{1,3}} \mathrm{~d}^{4} x \operatorname{tr}\left(\lambda^{2}\right)
$$

In order to extract the mass, the field needs to be canonically normalized. The 4D kinetic term is given by

$$
\mathrm{i} \tau_{\mathrm{D} 7} g_{s}^{-1} \int \mathrm{d}^{8} \xi \mathrm{e}^{\Phi} \sqrt{|\operatorname{det} \mathcal{M}|} \bar{\Theta} P_{-}^{\mathrm{D} 7} g^{\mu \nu} \Gamma_{\mu} \partial_{\nu} \Theta=\frac{\mathrm{i} \tau_{\mathrm{D} 7}}{2} \int_{\mathbb{R}^{1,3}} \mathrm{~d}^{4} x \operatorname{tr}(\lambda \not \partial \lambda) \int_{\Sigma_{4}} \mathrm{~d}^{4} x \sqrt{|\operatorname{det} \mathcal{M}|} h .
$$

The 4D gauge coupling which follows from dimensional reduction of the bosonic part of the $\mathrm{D} 7$ action and is identified with the visible sector $\mathrm{SU}(K)$ coupling is given by ${ }^{12}$

$$
\frac{1}{g_{\mathrm{vis}}^{2}}=\tau_{\mathrm{D} 7}\left(2 \pi \alpha^{\prime}\right)^{2} \int_{\Sigma_{4}} \mathrm{~d}^{4} x \sqrt{|\operatorname{det} \mathcal{M}|} h
$$

so the kinetic term can be expressed as

$$
\frac{1}{8 \pi^{2} \alpha^{2} g_{\text {vis }}^{2}} \int_{\mathbb{R}^{1,3}} \mathrm{~d}^{4} x \operatorname{tr}(\lambda \not \partial \lambda) .
$$

Canonically normalizing the field amounts to dividing by the prefactor of (3.31) so (3.27) gives a contribution to the gaugino mass

$$
\delta m_{1 / 2} \sim g_{s} M \varepsilon^{2 / 3} \tau_{\min }^{4} g_{\text {vis }}^{2} \mathcal{S} .
$$

In [27], the parameter $\mathcal{S}$ was related to the vacuum energy in the dual field theory

$$
\mathcal{S} \sim \mathcal{S}^{\mathrm{DKM}_{\varepsilon}}{ }^{-8 / 3} \sim\left(\frac{\Lambda_{\mathcal{S}}}{\Lambda_{\varepsilon}}\right)^{4},
$$

\footnotetext{
${ }^{12}$ Note that the value for $g_{\mathrm{vis}}$ is sensitive to the perturbation to the geometry, but this effect can be neglected to leading order in the perturbation.
} 
so that

$$
\delta m_{1 / 2} \sim g_{\mathrm{vis}}^{2} \lambda\left(\Lambda_{\varepsilon}\right) \frac{\Lambda_{\mathcal{S}}^{4}}{\Lambda_{\varepsilon}^{3}}\left(\left(\frac{m_{\chi}}{\Lambda_{\varepsilon}}\right)^{3 / 2}-1\right)^{2},
$$

where $\lambda\left(\Lambda_{\varepsilon}\right)=g_{s} M$ is the 't Hooft coupling of the hidden sector $\mathrm{SU}(M)$ in the far IR, $m_{\chi}=\mu^{2 / 3}$ is the messenger mass and $\Lambda_{\varepsilon}=\varepsilon^{2 / 3}$ is the confining scale.

Another contribution resulting from perturbing only the 3 -form flux potentially comes from

$$
\left.\operatorname{tr}\left\{\bar{\theta}\left(\mathcal{M}^{-1}\right)^{(\alpha \beta)} \Gamma_{\beta}\left(G_{M N P}^{*} \Gamma^{M N P} \Gamma_{\alpha}+\frac{1}{2} \Gamma_{\alpha}\left(G_{M N P}^{*}-G_{M N P}\right) \Gamma^{M N P}\right)\right] \theta\right\} .
$$

After some manipulation of the $\Gamma$-matrices, this can be written as

$$
-\operatorname{tr}\left\{\bar{\theta}\left(\frac{1}{2}\left(\mathcal{M}^{-1}\right)^{(\alpha \beta)} \gamma_{\alpha \beta}\left(G_{M N P}+G_{M N P}^{*}\right) \Gamma^{M N P}-6\left(\mathcal{M}^{-1}\right)^{(\alpha \beta)} \Gamma_{\alpha} \Gamma_{M N} G_{\beta}^{* M N}\right) \theta\right\}
$$

Since $\gamma$ and $\mathcal{M}^{-1}$ are unperturbed, they satisfy

$$
\left(\mathcal{M}^{-1}\right)^{(\alpha \beta)} \gamma_{\alpha \beta}=8-\frac{2^{2 / 3} \tau_{\min }^{2}}{3^{4 / 3} a_{0}}+\mathcal{O}\left(\tau_{\min }^{4}\right),
$$

where we have used the pullbacks presented in Appendix Q. Thus to leading order in $\tau_{\min }$, the first two terms of (3.36), which couple to the $(3,0)$ and $(0,3)$ parts of $G_{3}$ respectively, result in contributions to the gaugino mass that are parametrically the same as (3.34). The third term of (3.36) can be cast as

$$
\operatorname{tr}\left\{\bar{\theta} \frac{\partial x^{p}}{\partial \xi^{a}} \frac{\partial x^{q}}{\partial \xi^{b}}\left(\mathcal{M}^{-1}\right)^{(a b)} g^{m n} g^{s t} G_{p n t}^{*} \Gamma_{q} \Gamma_{m s} \theta\right\}
$$

where the D7-brane world-volumes are specified by $x^{M}=x^{M}\left(\xi^{\alpha}\right)$. Using the gaugino wavefunction and the relation between the warped and unwarped $\Gamma$-matrices, this becomes (up to combinatorial factors)

$$
\operatorname{tr}\left(\lambda^{2}\right) \frac{\partial x^{p}}{\partial \xi^{\alpha}} \frac{\partial x^{q}}{\partial \xi^{\beta}}\left(\tilde{\mathcal{M}}^{-1}\right)^{\alpha \beta} \tilde{g}^{m n} \tilde{g}^{s t} G_{p n t}^{*} \bar{\Omega}_{q m s} .
$$

where we have defined the "unwarped" NS-NS tensor

$$
\tilde{M}_{a b}=\tilde{\gamma}_{a b}+h^{-1 / 2} g_{s}^{1 / 2} \mathrm{e}^{-\Phi / 2} b_{a b}
$$

Because of the non-trivial embedding, this term is more difficult to compute. However, a similar computation was considered in 25] in the KT region where it was useful to introduce holomorphic 1-forms that are analogous to (B.14). Since the world-volumes of the D7-branes are specified by the holomorphic condition (2.3), the unperturbed induced metric is Hermitian. Similarly, the fact that the D7-branes are supersymmetric in the KS background implies that 
$b_{2}$ is $(1,1)$ 52, 53 and therefore $\mathcal{M}$ has non-vanishing values only for the components with one holomorphic and one anti-holomorphic index. Then (3.38) can be written

$$
\operatorname{tr}\left(\lambda^{2}\right) \frac{\partial Z^{I}}{\partial \zeta^{\sigma}} \frac{\partial \bar{Z}^{\bar{I}^{\prime}}}{\partial \bar{\zeta}^{\bar{\rho}}}\left(\tilde{\mathcal{M}}^{-1}\right)^{(\sigma \bar{\rho})} \tilde{g}^{J \bar{J}^{\prime}} \tilde{g}^{K \bar{K}^{\prime}} G_{I J K}^{*} \bar{\Omega}_{\bar{I}^{\prime} \bar{J}^{\prime} \bar{K}^{\prime}}
$$

where $I, J, K$ indicate the coordinates used in (B.14) and $\zeta$ are some complex coordinates on $\Sigma_{4}$ whose exact form we will not need. To leading order,

$$
\tilde{g}^{J \bar{J}^{\prime}} \tilde{g}^{K \bar{K}^{\prime}} G_{I J K}^{*} \bar{\Omega}_{\bar{I}^{\prime} \bar{J}^{\prime} \bar{K}^{\prime}} \sim \frac{M \alpha^{\prime} \mathcal{S}}{\varepsilon^{2 / 3}}\left(\begin{array}{ccc}
\tau^{-1} & \\
& \tau & \\
& & \tau^{-1}
\end{array}\right),
$$

where $I=1,2,3$ and where terms higher order in $\tau$ have been dropped.

To precisely calculate (3.41), we would need to transform from these coordinates to $Z^{I}$, taking into account the non-trivial pullback. However, since we are only interested in the leading parametric dependence, it will suffice to consider the component of the symmetrized $\mathcal{M}^{-1}$ which has the leading $\left(\tau-\tau_{\min }\right)$ and $\tau_{\text {min }}$ behavior. Using the pullbacks presented in $\square$, this component is

$$
\left(\tilde{\mathcal{M}}^{-1}\right)^{h_{2} h_{2}} \sim \frac{1}{\tau_{\min }\left(\tau-\tau_{\min }\right) \varepsilon^{4 / 3}} .
$$

One can show that in addition to complicated angular dependence, the coordinate transformation is parametrically effected by multiplication by $\tau_{\text {min }}^{2}$. Putting these together, we find that the leading order behavior is

$$
\frac{\partial x^{p}}{\partial \xi^{\alpha}} \frac{\partial x^{q}}{\partial \xi^{\beta}}\left(\tilde{\mathcal{M}}^{-1}\right)^{(\alpha \beta)} \tilde{g}^{m n} \tilde{g}^{s t} G_{p n t}^{*} \bar{\Omega}_{q m s} \sim \frac{M \alpha^{\prime}}{\varepsilon^{2}\left(\tau-\tau_{\min }\right)} .
$$

Comparing this to (3.19) which results in (3.34), we find that (3.44) contributes to the gaugino mass an amount that is parametrically the same as (3.34).

Further contributions due to perturbed 3-form flux possibly come from

$$
\operatorname{tr}\left\{\bar{\theta}\left(\mathcal{M}^{-1}\right)^{[\alpha \beta]} \Gamma_{\beta}\left(G_{M N P} \Gamma^{M N P} \Gamma_{\alpha}+\frac{1}{2} \Gamma_{\alpha}\left(G_{M N P}-G_{M N P}^{*}\right) \Gamma^{M N P}\right) \theta\right\} .
$$

Following similar steps that lead to (3.36), this becomes

$$
-\operatorname{tr}\left\{\bar{\theta}\left(\mathcal{M}^{-1}\right)^{[\alpha \beta]} \Gamma_{\beta}\left(\Gamma_{\alpha}\left(G_{M N P}+G_{M N P}^{*}\right) \Gamma^{M N P}-6 G_{\alpha N P} \Gamma^{N P}\right) \theta\right\} .
$$

Using the results in [50], when acting on the gaugino

$$
\left(\mathcal{M}^{-1}\right)^{a b} \Gamma_{a} \Gamma_{b} \theta=\left(\mathcal{M}^{-1}\right)^{b a} \Gamma_{a} \Gamma_{b} \theta .
$$

Since $\left(M^{-1}\right)^{[\mu \nu]}=0$, this implies (when acting on the gaugino)

$$
\left(\mathcal{M}^{-1}\right)^{[\alpha \beta]} \Gamma_{\alpha} \Gamma_{\beta} \theta=0 .
$$


Thus (3.45) becomes

$$
\operatorname{tr}\left\{\bar{\theta}\left(\mathcal{M}^{-1}\right)^{[\alpha \beta]} \Gamma_{\beta} G_{\alpha N P} \Gamma^{N P} \theta\right\} .
$$

This term involves a contraction similar to (B.14)

$$
\tilde{g}^{J \bar{J}^{\prime}} \tilde{g}^{K \bar{K}^{\prime}} G_{I J K} \bar{\Omega}_{\bar{I}^{\prime} \bar{J}^{\prime} \bar{K}^{\prime}} \sim \frac{M \alpha^{\prime} \mathcal{S}}{\varepsilon^{2 / 3}}\left(\begin{array}{ccc}
\tau^{-1} & & \\
& \tau & \\
& & \tau^{-1}
\end{array}\right) .
$$

The remaining indices are contracted with the anti-symmetric part of $\mathcal{M}^{-1}$. For the KS background, the most leading part is (using the pullbacks in Appendix @)

$$
\left(\mathcal{M}^{-1}\right)^{\left[\rho h_{2}\right]} \sim \frac{1}{\varepsilon^{4 / 3} \tau_{\min }^{1 / 2}\left(\tau-\tau_{\min }\right)^{1 / 2}} .
$$

Comparing to (3.43), this is subleading in $\left(\tau-\tau_{\min }\right)$ and $\tau_{\min }$ and thus will give a subleading contribution to the gaugino mass.

\subsubsection{Contributions from the perturbed metric}

We next need to take into account terms that result from perturbing the metric while leaving the flux unperturbed. The perturbations to the metric that are of the form $\delta g_{z \bar{z}}$ or $\delta g_{\bar{z} z}$ will not contribute to the gaugino mass since, as shown above, when the metric is Hermitian, the unperturbed $(2,1)$-component of $G_{3}$ does not contribute. However, as pointed out in [25], when the perturbed metric is no longer Hermitian with respect to the original complex structure, there are in general contributions to the gaugino mass from the components of the 3-form flux with mixed holomorphic and anti-holomorphic indices.

We again consider the first term in (3.2),

$$
\operatorname{tr}\left\{\bar{\theta} G_{M N P} \Gamma^{M N P} \theta\right\}=\operatorname{tr}\left(\lambda^{2}\right) \tilde{g}^{m n} \tilde{g}^{s r} \tilde{g}^{p q} G_{m n p} \eta^{T} \tilde{\Gamma}_{n r q} \eta
$$

If the metric is no longer Hermitian, then there is a contribution of the form

$$
\operatorname{tr}\left(\lambda^{2}\right) \tilde{g}^{\bar{i} \bar{i}^{\prime}} \tilde{g}^{j \bar{j}^{\prime}} \tilde{g}^{k \bar{k}^{\prime}} G_{\overline{i j k}} \bar{\Omega}_{\bar{i}^{\prime} \bar{j}^{\prime} \bar{k}^{\prime}}
$$

We could of course consider the contractions with even more non-Hermitian parts (i.e. terms with $\left.\tilde{g}^{\bar{i} \bar{i}^{\prime}} \tilde{g}^{j} \bar{j}^{\prime} \tilde{g}^{k \bar{k}^{\prime}}\right)$ but since the non-perturbed metric is Calabi-Yau, these are higher order in $\mathcal{S}$. Using the solution in Section 2.1, we find

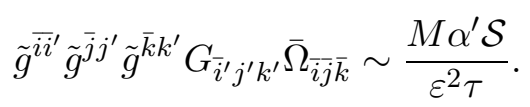

Comparing to (3.19), we see that this term contributes an amount that is parametrically the same as (3.34).

Since we are for now neglecting the change in $\mathcal{M}^{-1}$, the next group of terms 3.35) can again be written as (3.36) but now considering the 3 -form flux to be unperturbed and the 
non-Hermitian perturbations to the bulk metric. The first term in (3.36) again contributes parametrically the same as (3.52) since making use of (3.37) it is of a closely related form. The third term of (3.36) potentially has a contribution from the non-Hermitian perturbations of $g$,

$$
\operatorname{tr}\left(\lambda^{2}\right) \frac{\partial Z^{I}}{\partial \zeta^{\sigma}} \frac{\partial \bar{Z}^{\bar{I}^{\prime}}}{\partial \bar{\zeta}^{\bar{\rho}}}\left(\mathcal{M}^{-1}\right)^{\sigma \bar{\rho}} \tilde{g}^{\bar{J} \bar{J}^{\prime}} \tilde{g}^{K \bar{K}^{\prime}} G_{I \bar{J} K^{\prime}}^{*} \bar{\Omega}_{\bar{I}^{\prime} \bar{J}^{\prime} \bar{K}^{\prime}}
$$

where $\mathcal{M}^{-1}$ is unperturbed. Writing $G_{I \bar{J} K}^{*}=\left(G_{\bar{I} J \bar{K}}\right)^{*}$, we see that this is a coupling to the $(1,2)$ component of $G_{3}$. The unperturbed flux for the $\mathrm{KS}$ solution is purely ISD $(2,1)$, so this term vanishes and does not contribute to the gaugino mass. This argument which also applies to the second term of (3.36) $G_{M N P}^{*}$ when the metric is perturbed but the flux is not.

Next we consider contributions resulting from the perturbation of the symmetric part of $\mathcal{M}^{-1}$ in (3.36). Again, only the perturbations to the purely holomorphic and purely anti-holomorphic parts could possibly contribute to a gaugino mass (perturbations to the components of mixed type, for example $\delta \mathcal{M}_{z \bar{z}}$ do not contribute to the gaugino mass to leading order in $\mathcal{S})$. The first two terms couple to the $(3,0)$ parts of $G_{3}$ and $G_{3}^{*}$ and the third term couples to the $(1,2)$ part of $G_{3}$. Since the unperturbed flux $G_{3}(2,1)$, all of these contributions vanish to leading order in $\mathcal{S}$.

There could additionally be contributions from the purely holomorphic and purely antiholomorphic perturbations to the anti-symmetric part of $\mathcal{M}^{-1}$ in (3.46). The first two terms of (3.36) give a coupling to the $(0,3)$ and $(3,0)$ parts of $G_{3}$ which vanish. However, the third term gives a coupling to the $(2,1)$-component of $G_{3}$ which is non-vanishing in KS. To leading order in $\tau$, the contraction of the unperturbed fields gives

$$
\tilde{g}^{J \bar{J}^{\prime}} \tilde{g}^{K \bar{K}^{\prime}} G_{\bar{I} J K} \bar{\Omega}_{\bar{I}^{\prime} \bar{J}^{\prime} \bar{K}^{\prime}} \sim \frac{M \alpha^{\prime}}{\varepsilon^{2 / 3}}\left(\begin{array}{ccc}
1 & & \\
& \tau^{2} & \\
& & 1
\end{array}\right) .
$$

The remaining indices are again contracted with the anti-symmetric part of $\mathcal{M}^{-1}$. Since we are interested in only the parametric dependence, we consider the component of the antisymmetrized $\mathcal{M}^{-1}$ with the most singular dependence in $\tau_{\min }$ and $\left(\tau-\tau_{\min }\right)$, focusing on the part proportional to $\mathcal{S}$ (since the parts not proportional to $\mathcal{S}$ cannot contribute here). The leading component is (see Appendix C)

$$
\left(\tilde{M}^{-1}\right)^{\left[\rho h_{2}\right]} \sim \frac{\mathcal{S}}{\tau_{\min }^{7 / 2}\left(\tau-\tau_{\min }\right)^{1 / 2} \varepsilon^{4 / 3}}
$$

Taking into account the coordinate transformations, to leading order

$$
\sqrt{|\operatorname{det} \mathcal{M}|} \frac{\partial x^{p}}{\partial \xi^{\alpha}} \frac{\partial x^{q}}{\partial \xi^{\beta}}\left(\tilde{\mathcal{M}}^{-1}\right)^{[\alpha \beta]} \tilde{g}^{m n} \tilde{g}^{s t} G_{p n t} \bar{\Omega}_{q m s} \sim M \alpha^{\prime} \varepsilon^{2 / 3} \tau_{\min }^{2} \mathcal{S} .
$$

Comparing to (3.27) which yielded (3.34), we get the contribution to the gaugino mass

$$
\delta m_{1 / 2} \sim g_{s} M \varepsilon^{2 / 3} \tau_{\min }^{3} g_{\text {vis }}^{2} \mathcal{S}
$$


In terms of the parameters of the dual field theory,

$$
\delta m_{1 / 2} \sim g_{\mathrm{vis}}^{2} \lambda\left(\Lambda_{\varepsilon}\right) \frac{\Lambda_{\mathcal{S}}^{4}}{\Lambda_{\varepsilon}^{3}}\left(\left(\frac{m_{\chi}}{\Lambda_{\varepsilon}}\right)^{3 / 2}-1\right)^{3 / 2},
$$

which parametrically contributes more significantly than the previous contributions.

\subsubsection{Other 3-form contributions}

For each of the above terms, we have neglected the fact that the measure $e^{3 \Phi / 2} \sqrt{|\operatorname{det} \mathcal{M}|}$ and the $\Gamma$-matrices should also be modified in the new geometry. However, since we are working to first order in $\mathcal{S}$, perturbing the measure means to consider the Dirac-like operator (i.e. $\bar{\theta} \cdots \theta$ ) to be unperturbed. Since the unperturbed operator does not give a mass to the gaugino, perturbing the measure will not contribute to $m_{1 / 2}$ to linear order in $\mathcal{S}$.

Perturbations to the holomorphic $\Gamma$-matrices are of the form

$$
\delta \Gamma_{i} \sim a^{j}{ }_{i} \Gamma_{j}+b^{\bar{j}}{ }_{i} \Gamma_{\bar{j}}
$$

The perturbations proportional to the holomorphic $\Gamma$-matrices will not give any new contribution to the gaugino mass since the holomorphic $\Gamma$-matrices annihilate the gaugino. However, the terms proportional to the anti-holomorphic $\Gamma$-matrices will contribute through terms such as

$$
\bar{\theta} g^{i \bar{i}^{\prime}} g^{j \bar{j}^{\prime}} g^{k \bar{k}^{\prime}} G_{\bar{i}^{\prime} j k} \delta \Gamma_{i} \Gamma_{\bar{j}^{\prime}} \Gamma_{\bar{k}^{\prime}} \theta \sim b_{\ell}^{\bar{i}} \bar{\Omega}^{\widetilde{\ell j k}} G_{\overline{i j k}}^{-}
$$

where $b_{j}{ }^{\bar{i}}=g^{k \bar{i}} g_{j} b^{\bar{\ell}}{ }_{k}$. This term couples to the non-vanishing $(2,1)$ component of the KS 3 -form flux and is expected to parametrically contribute the same amount as previous contributions. Similar arguments apply when considering perturbations to the anti-holomorphic $\Gamma$-matrices.

In addition to the above effects, one must take into account the fact that the $\overline{\mathrm{D} 3}$-branes will interact with the D7-branes, though the consideration is very closely related to the above discussions. That is, $z_{4}=\mu$ is a volume minimizing condition in the KS geometry, but when we perturb the geometry, this condition will no longer hold. The world-volumes will be slightly perturbed so that the embedding is specified by

$$
\mathcal{F}\left(z_{i}, \bar{z}_{i} ; \mu, \varepsilon, \mathcal{S}\right)=0
$$

for some function $\mathcal{F}$. To leading order in perturbation theory, we can consider a stack of D7-branes satisfying (3.63) in the original KS geometry. With this new condition, in general the D7-branes will no longer have a complex structure that is compatible with that of the bulk geometry $^{13}$. For example, the pullback of a $(1,0)$-form will not in general be a $(1,0)$-form with respect to any world-volume complex structure. Since the existence of the gaugino mass depends on the Hodge types of the fluxes, this might result in a non-vanishing mass for the

\footnotetext{
${ }^{13}$ Indeed, the new world-volumes may not, in general, even admit a complex structure. However, relaxing the assumption that the world-volumes admits a complex structure will not effect the conclusion of this discussion.
} 
gaugino (which is simply the statement that if the D7-brane is not holomorphically embedded into the geometry, then it is not supersymmetric). However, the relative change in Hodge type is $\mathcal{O}(\mathcal{S})$. That is, if $w_{\sigma}$ are complex coordinates on the D7 world-volumes, then

$$
\mathrm{P}[\mathrm{d} z] \sim \mathrm{d} w+\mathcal{S} \mathrm{d} \bar{w}
$$

A possible gaugino mass could arise from the term ${ }^{14}$

$$
\left(\mathcal{M}^{-1}\right)^{(a b)} g^{m n} g^{s t} \bar{\theta} \Gamma_{a m s} G_{b n t}^{*} \theta \sim\left(\mathcal{M}^{-1}\right)^{(a b)} g^{m n} g^{s t} \bar{\Omega}_{a m s} G_{b n t}^{*}
$$

Since the complex structure of the world-volumes may be different than that of the bulk, this is generally of the form

$$
\left(\mathcal{M}^{-1}\right)^{(\sigma \bar{\rho})} g^{i \bar{i}^{\prime}} g^{j \bar{j}^{\prime}}\left(\bar{\Omega}_{\sigma \bar{i}^{\prime} \bar{j}^{\prime}} G_{\bar{\rho} i j}^{*}+\bar{\Omega}_{\bar{\rho} \bar{i}^{\prime} \bar{j}^{\prime}} G_{\sigma i j}^{*}\right),
$$

where we have chosen the world-volume complex structure such that the $\mathcal{M}$ is non-vanishing only for components with one holomorphic and one anti-holomorphic index. In the KS background however, both of these terms vanish. Considering the first term, since $\bar{\Omega}_{\sigma \bar{i}^{\prime} \bar{j}^{\prime}}$ has a change in the complex structure ( $\sigma$ is a holomorphic world-volume index but $\bar{\Omega}$ is $(0,3)$ in the bulk), it is proportional to $\mathcal{S}$. Therefore, the only part of $G_{\bar{\rho} i j}^{*}$ that contributes is such that the Hodge type is compatible with the bulk complex structure; that is, the part that contributes is the part of $G_{3}^{*}$ that is $(2,1)$ with respect to the bulk complex structure as well. Since the $(2,1)$ part of $G_{3}^{*}$ is essentially the $(1,2)$ part of $G_{3}$ and to this order in perturbation the flux is unperturbed, $G_{\bar{\rho} i j}^{*}=0$. Similarly, the second term couples to the $(3,0)$ and $(2,1)$ parts of $G_{3}^{*}$ (with respect to the bulk complex structure). Both of these vanish in KS, and so the second term vanishes as well.

The arguments for the vanishing of these terms were very similar to those for the perturbations to the symmetrized part of $\mathcal{M}$ considered in Section 3.1.2. An analogous argument for the anti-symmetric part of $\mathcal{M}^{-1}$ would show that there is a coupling to the bulk $(2,1)$ part of $G_{3}$ when the world-volumes are perturbed. Although it would be necessary to calculate $\mathcal{F}$ appearing in $(3.63)$ to calculate this exactly, we expect that it should be parametrically similar to $(3.60)$.

\subsection{Contributions from the 5 -form flux}

All of the above subsections focused on the contributions related to the 3 -form flux and were similar to discussions in [43] and [25]. However, in principle there could be additional contributions from other SUSY-breaking bulk fields.

For example, there is the possibility of a mass arising from the 5-form flux. In the SUSY case, the 5 -form flux is related to the warp factor so we must consider the spin connection as

\footnotetext{
${ }^{14}$ To this order in perturbation theory, the gaugino wavefunction is unperturbed so it is annihilated by $\Gamma$-matrices that are holomorphic with respect to the KS complex structure.
} 
well. The action contains,

$\mathrm{i} \tau_{\mathrm{D} 7} g_{s}^{-1} \int \mathrm{d}^{8} \xi \mathrm{e}^{\Phi} \sqrt{|\operatorname{det} \mathcal{M}|} \operatorname{tr}\left\{\bar{\Theta} P_{-}^{\mathrm{D} 7}\left(\hat{\mathcal{M}}^{-1}\right)^{\alpha \beta} \Gamma_{\beta}\left(\nabla_{\alpha}+\frac{g_{s}}{16 \cdot 5 !} \tilde{F}_{N P Q R T} \Gamma^{N P Q R T} \Gamma_{\alpha}\left(\mathrm{i} \sigma_{2}\right)\right) \Theta\right\}$.

$\nabla_{\alpha}$ is the pullback of the covariant derivative which has components

$$
\begin{aligned}
\nabla_{\mu} & =\partial_{\mu}-\frac{1}{8} \Gamma_{\mu} \not \partial \log h, \\
\nabla_{m} & =\tilde{\nabla}_{m}+\frac{1}{8} \Gamma_{m} \not \partial \log h-\frac{1}{8} \partial_{m} \log h,
\end{aligned}
$$

where $\tilde{\nabla}$ is the covariant derivative with respect to the unwarped $6 \mathrm{D}$ metric which in this subsection we take to be unperturbed. Following [50], and using the $\kappa$-fixing condition (3.7), this becomes (when acting on the gaugino)

$$
\begin{aligned}
\frac{\mathrm{i} \tau_{\mathrm{D} 7}}{2 g_{s}} \int \mathrm{d}^{8} \xi \mathrm{e}^{\Phi} \sqrt{|\operatorname{det} \mathcal{M}|} \operatorname{tr}\left\{\overline { \theta } \left[\left(\mathcal{M}^{-1}\right)^{a b} \Gamma_{a}\left(\tilde{\nabla}_{b}-\frac{1}{8} \partial_{b} \log h\right)\right.\right. \\
+\frac{\left(g_{s} M \alpha^{\prime}\right)^{2}}{16} \ell(\tau) \frac{\sqrt{p}}{\sqrt{u} s q h}\left(\mathcal{M}^{-1}\right)^{a b}\left(\partial_{a} \tau\right) \Gamma_{b}-\frac{1}{2}\left(1-\frac{1}{4}\left(\mathcal{M}^{-1}\right)^{a b} \Gamma_{a} \Gamma_{b}\right) \not \partial \log h \\
\left.\left.+\frac{\left(g_{s} M \alpha^{\prime}\right)^{2}}{8} \ell(\tau) \frac{\sqrt{p}}{\sqrt{u} s q h}\left(1-\frac{1}{4}\left(\mathcal{M}^{-1}\right)^{a b} \Gamma_{b} \Gamma_{a}\right) \Gamma^{\tau}\right] \theta\right\}
\end{aligned}
$$

where we have omitted the $4 \mathrm{D}$ kinetic term since it does not contribute to a mass term. Since $\theta$ is a Majorana-Weyl spinor, any bilinear $\bar{\theta} \Gamma_{M} \theta$ vanishes. Therefore, since the gaugino wavefunction behaves as $h^{3 / 8} \eta$ where $\eta$ is covariantly constant with respect to $\tilde{\nabla}$, (3.70) becomes

$$
\frac{\mathrm{i} \tau_{\mathrm{D} 7}}{16 g_{s}} \int \mathrm{d}^{8} \xi \mathrm{e}^{\Phi} \sqrt{|\operatorname{det} \mathcal{M}|} \operatorname{tr}\left\{\bar{\theta}\left(\mathcal{M}^{-1}\right)^{a b}\left[\Gamma_{a} \Gamma_{b} \not \partial \log h-\frac{\left(g_{s} M \alpha^{\prime}\right)^{2}}{4} \ell(\tau) \frac{\sqrt{p}}{\sqrt{u} s q h} \Gamma_{b} \Gamma_{a} \Gamma^{\tau}\right] \theta\right\} .
$$

Following similar arguments for the 3-form flux above, to obtain the contribution to the gaugino mass to linear order in $\mathcal{S}$, we consider perturbations to one field at a time. Perturbations to $h, \ell$, or any of the metric functions $p, u$, or $s$ do not give a contribution as the unperturbed $\mathcal{M}^{-1}$ has non-vanishing elements only for components with one holomorphic and one anti-holomorphic index so that

$$
\bar{\theta}\left(\mathcal{M}^{-1}\right)^{a b} \Gamma_{a} \Gamma_{b} \Gamma^{\tau} \theta
$$

consists of mixed holomorphic and anti-holomorphic $\Gamma$-matrices. A similar argument applies if we consider perturbing the measure $\mathrm{e}^{\Phi} \sqrt{|\operatorname{det} \mathcal{M}|}$.

The next potential contribution is from considering the perturbation to $\mathcal{M}^{-1}$. Since the perturbed $\mathcal{M}^{-1}$ contains pieces that are non-Hermitian, this may a priori contribute to the gaugino mass. The remaining fields are not perturbed from their KS values which satisfy

$$
h^{\prime}(\tau)=-\frac{\left(g_{s} M \alpha^{\prime}\right)^{2}}{4} \ell \frac{\sqrt{p}}{\sqrt{u} s q}
$$


so that (3.71) gives

$$
\frac{\mathrm{i} \tau_{\mathrm{D} 7}}{16} \int \mathrm{d}^{8} \xi \sqrt{|\operatorname{det} \mathcal{M}|} \operatorname{tr}\left\{\bar{\theta}\left(\mathcal{M}^{-1}\right)^{a b}\left\{\Gamma_{a}, \Gamma_{b}\right\} \not \partial \log h \theta\right\}
$$

Using the Clifford algebra the term in the trace is

$$
\left(\mathcal{M}^{-1}\right)^{a b} \gamma_{a b} \bar{\theta} \not \partial \log h \theta=0,
$$

where we have again used the fact that $\bar{\theta} \Gamma_{M} \theta=0$. Perturbations to the $\Gamma$-matrices will give the same form (3.75) except $\gamma$, rather than $\mathcal{M}^{-1}$ is perturbed, and so the term will also vanish.

Similar to the consideration of the 3 -form fluxes, we must also consider the effect of the deformation of the world-volumes. However, for the 3-form fluxes the important aspect was the change in complex structure as a result of the pullback. In this case, the complex structure of the pullbacks of the 5-form flux and the spin connection are not important for arguing for the vanishing of the mass. Therefore, considering the effect of the perturbation of the world-volumes is equivalent to considering perturbations to the fields and all of these contributions vanish.

\subsection{Contributions from the perturbed spin connection}

Additional contributions could potentially arise from the perturbed spin connection. The $6 \mathrm{D}$ manifold is perturbed from the deformed conifold geometry so that it is no longer conformally Calabi-Yau. Contained within the D7 Dirac-like action is the term

$$
\frac{\mathrm{i} \tau_{\mathrm{D} 7}}{2 g_{s}} \int \mathrm{d}^{8} \xi \operatorname{tr}\left\{\bar{\theta}\left(\mathcal{M}^{-1}\right)^{a b} \Gamma_{a} \tilde{\nabla}_{b} \theta\right\}
$$

where $\tilde{\nabla}$ is the pullback of the covariant derivative with respect to the unwarped $6 \mathrm{D}$ metric. When $\tilde{\nabla}$ is unperturbed, the fact that $\eta$ is covariantly constant causes this term to automatically vanish. Therefore, in considering non-vanishing contributions, we need only consider perturbations to $\tilde{\nabla}$. $\tilde{\nabla}$ is given by

$$
\tilde{\nabla}_{a}=\partial_{a}+\frac{1}{4} \tilde{\omega} \frac{M N}{a} \Gamma_{\underline{M N}}
$$

where $\tilde{\omega}$ is the spin connection built from the unwarped 6D metric. Perturbations to the spin connection then give the contribution

$$
\left(M^{-1}\right)^{a b} g^{m n} g^{s t} \delta \tilde{\omega}_{a m s} \bar{\Omega}_{b n t},
$$

where we only consider the perturbations to $\omega$, the unperturbed part being cancelled by the derivative $\partial_{a}$. A detailed calculation shows that this contraction vanishes for the isometrypreserving perturbation considered here. Some terms in the perturbed spin connection have the wrong Hodge type to contract with $\bar{\Omega}$. The sum over the remaining terms vanish based 
on the symmetries of $\delta \tilde{\omega}$. Thus there are no contributions to the visible sector gaugino mass coming from considering the perturbations to the $6 \mathrm{D}$ unwarped spin connection.

The components $\nabla_{\mu}$ are also perturbed in this geometry. However, since this geometry is unperturbed from Minkowski space, the only perturbation to the covariant derivative comes from the perturbations to the warp factor which were considered in Section 3.2 .

\section{Conclusion}

In this paper, we have used to the language of the gauge-gravity correspondence to consider the effects the effects of strong coupling dynamics on a relative of semi-direct gauge mediation. In particular, we examined the holographic gauge mediation scenario of 25] where the hidden sector is a cascading $\mathrm{SU}(N+M) \times \mathrm{SU}(N)$ gauge theory, but considered the regime where the messenger mass $m_{\chi}$ was comparable (and in fact very close to) the confinement scale $\Lambda_{\varepsilon}$. In the gravity dual, this required use of one of the solutions presented in [26] which described the influence of an $\overline{\mathrm{D} 3}$ on the near-tip geometry of the warped deformed conifold. The confining dynamics of the strongly coupled gauge theory breaks the $R$-symmetry to $\mathbb{Z}_{2}$, which allows the gaugino to get a mass from physics above $m_{\chi}$. To leading order in the SUSY-breaking order parameter

$$
\delta m_{1 / 2} \sim g_{\mathrm{vis}}^{2} \lambda\left(\Lambda_{\varepsilon}\right) \frac{\Lambda_{\mathcal{S}}^{4}}{\Lambda_{\varepsilon}^{3}}\left(\left(\frac{m_{\chi}}{\Lambda_{\varepsilon}}\right)^{3 / 2}-1\right)^{3 / 2},
$$

where $\Lambda_{\varepsilon}$ is the hidden sector confining scale, $\Lambda_{\mathcal{S}}$ is the vacuum energy, $m_{\chi}$ is the messenger mass, $g_{\text {vis }}$ is the visible sector gauge coupling, and $\lambda\left(\Lambda_{\varepsilon}\right)$ is the hidden sector 't Hooft coupling evaluated at the scale $\Lambda_{\varepsilon}$ (where the cascade has ended so the gauge group is the simple group $\mathrm{SU}(M))$. From the many possible terms that a priori could have given rise to a non-vanishing contributions, the only non-vanishing contributions come from the 3-form flux on the gravity side of the duality.

There are additional contributions from physics below the messenger mass. In particular, $\chi$ and $\tilde{\chi}$ bind into weakly interacting mesons and the spectrum contains mesons with masses below $m_{\chi}$. For $m_{\chi} \gg \Lambda_{\varepsilon}$, this contribution was calculated in 25] resulting in (2.5). Although we did not calculate the contribution from the mesons in this geometry, the mesons are weakly coupled in the large 't Hooft coupling limit while $\chi$ and $\tilde{\chi}$ are strongly coupled. Thus we expect the contribution to the gaugino mass from any one meson to be highly suppressed by 't Hooft coupling compared to the contributions from $\chi$.

The 't Hooft enhancement of (4.1) is quite different than the leading order contribution in the regime $m_{\chi} \gg \Lambda_{\varepsilon}$. In this regime, considered in [25], the large $R$-symmetry at high energies suppresses contributions to the gaugino mass from physics above the messenger mass $m_{\chi}$ and the leading order contribution comes from the 't Hooft suppressed interactions of mesonic bound states of the messenger quarks ${ }^{15}$. The fact that meson messengers are weakly coupled

\footnotetext{
${ }^{15}$ Although the contribution to $m_{1 / 2}$ from any single meson is ' $t$ Hooft suppressed, the sum of all contributions could be comparable to (4.1).
} 
in the 't Hooft limit required the authors of [25] to use a combination of perturbative field theory and holographic techniques. In contrast, the reduced amount of $R$-symmetry allowed us to compute the leading order contribution to the gaugino mass using only holography as the effective degrees of freedom are strongly coupled messengers.

Identification of the SUSY-breaking state in the dual gauge theory relies on the large radius behavior of the bulk gravitational fields. Since the solution used here is a small $\tau$ expansion, it is not useful for such an analysis. However, even using the large radius solution, it is not clear how to make the identification of the state in terms of dominant $F$-term or $D$-term breaking [27] and indeed at strong coupling the distinction may not be sharp (though the weakly coupled mesonic states discussed in [25] do feel effective $F$-terms). However, to leading order in $\tau_{\min }$, the contribution (4.1) can expressed as

$$
\delta m_{1 / 2} \sim g_{\mathrm{vis}}^{2} \lambda\left(\Lambda_{\varepsilon}\right) \frac{\Lambda_{\mathcal{S}}^{4}}{m_{\chi}^{3}}\left(\left(\frac{m_{\chi}}{\Lambda_{\varepsilon}}\right)^{3 / 2}-1\right)^{3 / 2} .
$$

This suggests that there is some $F$-term component to the SUSY-breaking breaking given by

$$
F=\sqrt{\lambda\left(\Lambda_{\varepsilon}\right)} \Lambda_{\mathcal{S}}^{2}
$$

The contribution to the gaugino mass from physics above $m_{\chi}$ is then

$$
\delta m_{1 / 2} \sim g_{\mathrm{vis}}^{2} \frac{F^{2}}{m_{\chi}^{3}}\left(\left(\frac{m_{\chi}}{\Lambda_{\varepsilon}}\right)^{3 / 2}-1\right)^{3 / 2} .
$$

The fact that the gaugino mass occurs at higher order in $F$ is similar to other examples of semi-direct gauge mediation where $m_{1 / 2}$ vanishes to leading order in $F$ [19, 25, 54].

This contribution to the gaugino mass naïvely vanishes for $m_{\chi}=\Lambda_{\varepsilon}$. We emphasize however that at this point the supergravity description breaks down since the D7s reach the curvature singularity where stringy effects are important and there may be important corrections to (4.2). However, the fact that it decreases with $\tau_{\min }$ may not be surprising. The statement that the integral is dominated near $\tau_{\text {min }}$ corresponds to the statement on the field theory side that the dominant contribution to the gaugino mass is from physics near $m_{\chi}$. Decreasing $\tau_{\min }$ corresponds to taking $m_{\chi}$ closer to $\Lambda_{\varepsilon}$ and so for smaller $\tau_{\min }$, the integral is dominated by physics at lower scales. Since the effective 't Hooft coupling decreases as the scale decreases, heuristically one might expect that this contribution to the gaugino mass also decreases.

The contribution (4.1) is a result of a calculation of a soft SUSY-breaking term that, unlike many other examples, at no point required the assumption of weak coupling in messenger or visible sectors aside from the gauge coupling to the standard model. Because the solutions presented in [26] were given only as a power series in $\tau$, it was not possible to find an exact expression for the gaugino mass. However, we emphasize that this difficulty is a very distinct difficulty from that usually faced by strong coupling in that one could in principle use the 
gauge-gravity correspondence to find an exact result in the limit of large 't Hooft coupling. In contrast, without holography it is not clear how even to perform this calculation, even in principle.

The messenger mass parameter $\mu$ has unit $R$-charge, so one would expect that for $\mu \neq 0$, even in the regime $m_{\chi} \gg \Lambda_{\varepsilon}$, there would be a contribution to $m_{1 / 2}$ from physics at all scales. However, for energies above $m_{\chi}$, the messenger quark $\chi$ is no longer integrated out of the effective field theory and the $R$-symmetry breaking effects are suppressed such that contributions to the gaugino mass occur only at subleading order in $\Lambda_{\mathcal{S}}$. At least as far as the gaugino mass is concerned, the $R$-symmetry breaking effect of a non-vanishing $\mu$ is less important than the $R$-symmetry breaking effect of confinement, though it would be worthwhile to develop a clearer picture. One possible step in this direction would be to take into account the back reaction of the D7-branes, essentially moving away from the quenched approximation in the field theory. For general $\mu$, such a back reaction would break the symmetry of the solution that is dual to the field theory $R$-symmetry.

It is well known that for theories of semi-direct or direct gauge mediation in which the hidden sector has large rank, one typically runs into a problem of visible sector Landau poles. Even in the regime of $m_{\chi} \gg \Lambda_{\varepsilon}$ discussed in [25], avoidance of visible sector Landau poles forced $m_{\chi}$ to be large. However, it was also suggested in [25] that the problem may be avoided by orbifolding the geometry. Although such a method might be needed to achieve realistic soft terms, we defer such analysis to future work.

An additional interesting future direction would be to holographically realize the visible sector matter fields as well. In the model of [25], the matter fields are taken to be elementary fields, living on the UV cutoff with other soft terms resulting from gaugino mediation. A more complete holographic realization of gauge mediation could involve a more detailed model in which the supersymmetric standard model (or some extension) is realized on a network of intersecting D7 branes carrying non-vanishing world-volume flux.

\section{Acknowledgments}

We would like to thank H.-Y. Chen, G. Kane, I.-W. Kim, F. Marchesano, P. Ouyang, X. Tata, A. Uranga, and L.-T. Wang for discussions and comments. PM and GS also thank T. Liu for collaboration and preliminary discussions on this and related topics. We would like to thank the Institute for Advanced Study and the Hong Kong Institute for Advanced Study, Hong Kong University of Science and Technology for hospitality and support. PM and GS also thank the Standford Institute for Theoretical Physics and SLAC for hospitality while some preliminary discussions were held. YS was supported by the Nishina Memorial Foundation. PM and GS were supported in part by NSF CAREER Award No. Phy-0348093, DOE grant DE-FG-02-95ER40896, a Cottrell Scholar Award from Research Corporation, a Vilas Associate Award from the University of Wisconsin, and a John Simon Guggenheim Memorial Foundation Fellowship. GS also would like to acknowledge support from the Ambrose Monell Foundation during his stay at the Institute for Advanced Study. 


\begin{tabular}{|c|c|}
\hline label & direction \\
\hline \hline$M, N$ & any direction \\
$\mu, \nu$ & 4D Minkowski \\
$m, n$ & radial and internal angular \\
$\alpha, \beta$ & D7 world-volume coordinate \\
$a, b$ & $\mathrm{D} 7$ world-volume coordinate: radial or internal angular \\
$i, j$ & complex coordinate $z_{i}$ defined in $(\overline{\mathrm{B} .1})$ \\
$I, J$ & complex coordinates $Z_{I}$ defined in (B.14) \\
$\sigma, \rho$ & complex D7 world-volume coordinates \\
\hline
\end{tabular}

Table 1: Index conventions. Exceptions should be clear from context. An index with a tilde (e.g. $\tilde{\alpha})$ indicates an index raised with the unwarped metric.

\section{A. Conventions}

Our index notation is summarized in Table 1 .

We work in the type IIB supergravity limit of string theory where the low energy effective action in the $10 \mathrm{D}$ Einstein frame is 55

$$
\begin{aligned}
& S_{\mathrm{IIB}}=S_{\mathrm{NS}}+S_{\mathrm{R}}+S_{\mathrm{CS}}, \\
& S_{\mathrm{IIB}}^{\mathrm{NS}}=\frac{1}{2 \kappa_{10}^{2}} \int \mathrm{d}^{10} x \sqrt{-\operatorname{det}(g)}\left[R-\frac{1}{2} \partial_{M} \Phi \partial^{M} \Phi-\frac{g_{s}}{2 \cdot 3 !} \mathrm{e}^{-\Phi} H_{3}^{2}\right], \\
& S_{\mathrm{IIB}}^{\mathrm{R}}=-\frac{1}{4 \kappa_{10}^{2}} \int \mathrm{d}^{10} x \sqrt{-\operatorname{det}(g)}\left[\mathrm{e}^{2 \Phi} \partial_{M} C \partial^{M} C+\frac{g_{s} \mathrm{e}^{\Phi}}{3 !} \tilde{F}_{3}^{2}+\frac{g_{s}^{2}}{2 \cdot 5 !} \tilde{F}_{5}^{2}\right], \\
& S_{\mathrm{IIB}}^{\mathrm{CS}}=\frac{g_{s}^{2}}{4 \kappa_{10}^{2}} \int C_{4} \wedge H_{3} \wedge F_{3},
\end{aligned}
$$

where in terms of the R-R potentials $C, C_{2}$, and $C_{4}$ and the NS-NS potential $B_{2}$,

$$
\tilde{F}_{3}=\mathrm{d} C_{2}-C H_{3}, \quad \tilde{F}_{5}=\mathrm{d} C_{4}+B_{2} \wedge \mathrm{d} C_{2} .
$$

$R$ is the Ricci scalar built from the metric $g$ and $\Phi$ is the dilaton such that $\langle\Phi\rangle=\log g_{s}$. The self-duality of the 5 -form field strength is imposed at the level of the equations of motion and we write

$$
F_{5}=\left(1+*_{10}\right) \mathcal{F}_{5}
$$

where $*_{10}$ is the Hodge star built from $g$. The gravitational coupling is $2 \kappa_{10}^{2}=(2 \pi)^{7} \alpha^{\prime 4} g_{s}^{2}$ and the Einstein frame metric $g_{M N}$ is related to the string frame metric $g_{M N}^{s}$ by the Weyl transformation $g_{M N}=g_{s}^{1 / 2} \mathrm{e}^{-\Phi / 2} g_{M N}^{s}$.

In the Einstein frame, the action for a $\mathrm{D} p$ brane is

$$
S_{\mathrm{D} p}=S_{\mathrm{D} p}^{\mathrm{DBI}}+S_{\mathrm{D} p}^{\mathrm{CS}}+S_{\mathrm{D} p}^{\mathrm{F}}
$$


where the bosonic part is,

$$
\begin{aligned}
S_{\mathrm{D} p}^{\mathrm{DBI}} & =-\tau_{\mathrm{D} p} g_{s}^{-\left(\frac{p-3}{4}\right) \Phi} \int \mathrm{d}^{p+1} \xi \mathrm{e}^{-\frac{(p-3)}{4}} \sqrt{\left|\operatorname{det}\left(\mathcal{M}_{\alpha \beta}\right)\right|} \\
S_{\mathrm{D} p}^{\mathrm{CS}} & =\tau_{\mathrm{D} p} \int \mathrm{P}\left[\sum_{n} C_{n} \wedge \mathrm{e}^{-B_{2}}\right] \wedge \mathrm{e}^{2 \pi \alpha^{\prime} f_{2}}
\end{aligned}
$$

with

$$
\mathcal{M}_{\alpha \beta}=\gamma_{\alpha \beta}+g_{s}^{1 / 2} \mathrm{e}^{-\Phi / 2} b_{\alpha \beta}+g_{s}^{1 / 2} \mathrm{e}^{-\Phi / 2}\left(2 \pi \alpha^{\prime}\right) f_{\alpha \beta}
$$

where $\gamma_{\alpha \beta}$ and $b_{\alpha \beta}$ are the pullbacks of the metric and the NS-NS 2-form potential onto the world-volume of the brane, $f_{2}=\mathrm{d} A_{1}$ is the field strength for the vector potential living on the world-volume, $\mathrm{P}[\cdot]$ indicates a pullback, and the tension of a $\mathrm{D} p$-brane satisfies $\tau_{\mathrm{D} p}^{-1}=$ $(2 \pi)^{p} \alpha^{\prime(p+1) / 4} g_{s}^{-1}$. $\overline{\mathrm{D} p}$-branes are distinguished by an overall sign in front of the ChernSimons piece. Although the generalization to the non-Abelian case is known [56], it is not needed for our purposes.

The fermionic action for a $\mathrm{D} p$ brane can be expanded out to quadratic order to give a Dirac-like action [47]. In the Einstein frame, this is given by ${ }^{16}$

$$
S_{\mathrm{D} p}^{(\mathrm{F})}=\mathrm{i} \tau_{\mathrm{D} p} g_{s}^{-\left(\frac{p-3}{4}\right)} \int \mathrm{d}^{8} \xi \mathrm{e}^{\left(\frac{p-3}{4}\right) \Phi} \sqrt{\operatorname{det}(\mathcal{M})} \bar{\Theta} P_{-}^{\mathrm{D} p}\left[\left(\mathcal{M}^{-1}\right)^{\alpha \beta} \Gamma_{\beta}\left(\mathcal{D}_{\alpha}+\frac{1}{4} \Gamma_{\alpha} \mathcal{O}\right)-\mathcal{O}\right] \Theta
$$

where $\mathcal{D}_{\alpha}$ and $\mathcal{O}$ are the pullbacks of operators involved in the Einstein-frame SUSY transformations of the gravitino and dilatino,

$$
\delta \Psi_{M}=\mathcal{D}_{M} \epsilon, \quad \delta \lambda_{\Phi}=\mathcal{O} \epsilon,
$$

where these are related to string frame fields by

$$
\epsilon=g_{s}^{1 / 8} \mathrm{e}^{-\Phi / 8} \epsilon^{s}, \quad \lambda_{\Phi}=g_{s}^{-1 / 8} \mathrm{e}^{\Phi / 8} \lambda^{s}, \quad \Psi_{M}=g_{s}^{1 / 8} \mathrm{e}^{-\Phi / 8}\left(\Psi_{M}^{s}-\frac{1}{4} \Gamma_{M}^{\mathrm{S}} \lambda_{\Phi}^{s}\right),
$$

and similarly $\Theta=g_{s}^{1 / 8} \mathrm{e}^{-\Phi / 8} \Theta^{s}$. $\Theta=\left(\theta_{1} \theta_{2}\right)^{\mathrm{T}}$ is a doublet of 10D Majorana-Weyl spinors satisfying $\Gamma_{(10)} \theta_{i}=\theta_{i}$ where $\Gamma_{(10)}$ is the $10 \mathrm{D}$ chirality operator. $\bar{\Theta}$ is defined by

$$
\bar{\Theta}=\left(\bar{\theta}_{1} \bar{\theta}_{2}\right)
$$

Following [47], we take the $\Gamma$-matrices to be real implying $\bar{\theta}=\theta^{T} \Gamma^{0}$ where underlined indices denote "flat" $\Gamma$-matrices. $P_{-}^{\mathrm{D} p}$ is a projection operator defined by

$$
P_{ \pm}^{\mathrm{D} p}=\frac{1}{2}\left(1 \pm \Gamma_{\mathrm{D} p}\right)=\frac{1}{2}\left(\begin{array}{cc}
1 & \pm \breve{\Gamma}_{\mathrm{D} p}^{-1} \\
\pm \breve{\Gamma}_{\mathrm{D} p} & 1
\end{array}\right)
$$

with

$$
\breve{\Gamma}_{\mathrm{D} p}=\mathrm{i}^{(p-2)(p-3)} \Gamma_{\mathrm{D} p}^{(0)} \Lambda(\mathcal{F})
$$

\footnotetext{
${ }^{16}$ The conventions here differ from those in [47, 50] by an opposite sign for $H_{3}$.
} 
where $\Gamma_{\mathrm{D} p}^{(0)}$ is given by

$$
\Gamma_{\mathrm{D} p}^{(0)}=\frac{1}{(p+1) ! \sqrt{-\operatorname{det}(\gamma)}} \epsilon_{\alpha_{1} \ldots \alpha_{p+1}} \Gamma^{\alpha_{1} \ldots \alpha_{p+1}},
$$

and

$$
\Lambda(\mathcal{F})=\frac{\sqrt{\operatorname{det}(\gamma)}}{\sqrt{\operatorname{det}(M)}} \sum_{q} \frac{\left(g_{s} \mathrm{e}^{-\Phi / 2}\right)^{q / 2}}{q ! 2^{q}} \mathcal{F}_{\alpha_{1} \alpha_{2}} \cdots \mathcal{F}_{a_{2 q-1} \alpha_{2 q}} \Gamma^{\alpha_{1} \cdots \alpha_{2 q}},
$$

where $\mathcal{F}_{2}=b_{2}+2 \pi \alpha^{\prime} f_{2}$. In terms of the usual $(2 k+2)$-dimensional chirality matrix

$$
\Gamma_{(2 k+2)}=\mathrm{i}^{k} \Gamma^{0 \cdots 2 k+1},
$$

where $\Gamma \underline{M}$ is a flat $\Gamma$-matrix, we have

$$
\mathrm{i}^{(p-2)(p-3)} \Gamma_{\mathrm{D} p}^{(0)}=\mathrm{i}^{(p-1) / 2} \Gamma_{(p+1)} .
$$

Except for $\Gamma_{\mathrm{D} p}$, all $\Gamma$-matrices act as identity on the doublet space,

$$
\Gamma_{M} \Theta=\left(\begin{array}{c}
\Gamma_{M} \theta_{1} \\
\Gamma_{M} \theta_{2}
\end{array}\right)
$$

In IIB,

$$
\hat{\mathcal{M}}_{\alpha \beta}=\gamma_{\alpha \beta}+g_{s}^{1 / 2} \mathrm{e}^{-\Phi / 2} \mathcal{F}_{\alpha \beta} \Gamma_{(10)} \otimes \sigma_{3}
$$

where the Pauli matrices act on the doublet space so that we can effectively write

$$
\bar{\Theta} \hat{\mathcal{M}}_{\alpha \beta}=\bar{\Theta}\left(\begin{array}{cc}
\mathcal{M}_{\beta \alpha} & \\
& \mathcal{M}_{\alpha \beta}
\end{array}\right) \text {. }
$$

In the IIB Einstein frame,

$$
\begin{gathered}
\mathcal{O}=\frac{1}{2} \Gamma^{M} \partial_{M} \Phi-\frac{1}{2} \mathrm{e}^{\Phi} \Gamma^{M} \partial_{M} C\left(\mathrm{i} \sigma_{2}\right)-\frac{1}{4} g_{s}^{1 / 2} \mathrm{e}^{\Phi / 2} \mathcal{G}_{3}^{+}, \\
\mathcal{D}_{M}=\nabla_{M}+\frac{1}{4} \mathrm{e}^{\Phi} \partial_{M} C\left(\mathrm{i} \sigma_{2}\right)+\frac{1}{8} \mathrm{e}^{\Phi / 2} g_{s}^{1 / 2}\left(\mathcal{G}_{3}^{-} \Gamma_{M}+\frac{1}{2} \Gamma_{M} \mathcal{G}_{3}^{-}\right) \\
+\frac{1}{16 \cdot 5 !} g_{s} \tilde{F}_{N P Q R T} \Gamma^{N P Q R T} \Gamma_{M}\left(\mathrm{i} \sigma_{2}\right),
\end{gathered}
$$

where

$$
\mathcal{G}_{3}^{ \pm}=\frac{1}{3 !}\left(\tilde{F}_{M N P} \sigma_{1} \pm \mathrm{e}^{-\Phi} H_{M N P} \sigma_{3}\right) \Gamma^{M N P} .
$$

$\sigma_{i=1,2,3}$ are the usual Pauli matrices

$$
\sigma_{1}=\left(\begin{array}{ll}
0 & 1 \\
1 & 0
\end{array}\right), \quad \sigma_{2}=\left(\begin{array}{cc}
0 & -\mathrm{i} \\
\mathrm{i} & 0
\end{array}\right), \quad \sigma_{3}=\left(\begin{array}{cc}
1 & 0 \\
0 & -1
\end{array}\right) .
$$

The non-Abelian generalization of the action in [47] is not known. However, to leading order in $\alpha^{\prime}$, and as long as the transverse fluctuations are suppressed, the non-Abelian action should result from promoting $\theta$ to an adjoint-valued field and the regular derivative to a gauge covariant derivative, and tracing over gauge indices. 


\section{B. Deformed conifold geometry}

Here we briefly review the geometry of the conifold and its deformation following closely the discussion in [36] (though see also [57]). The deformed conifold can be described as the locus of points satisfying

$$
\sum_{i=1}^{4} z_{i}^{2}=\varepsilon^{2},
$$

and the singular conifold is recovered for $\varepsilon=0$. Eq. ( $(\overline{B .1})$ is invariant under the $\mathbb{Z}_{2}$ transformation $z_{i} \rightarrow-z_{i}$ and the $\mathrm{SO}(4)$ transformation $z_{i} \rightarrow O_{i j} z_{j}$. The radial coordinates $\tau$ and $r$ are defined by

$$
z_{i} \bar{z}_{i}=\varepsilon^{2} \cosh \tau=r^{3} .
$$

The angular space is an $S^{3}$ fibered over an $S^{2}$ and is frequently written in terms of angular coordinates $\theta_{i=1,2} \in[0, \pi], \phi_{i=1,2} \in[0,2 \pi)$, and $\psi \in[0,4 \pi)$ related to the $z_{i}$ by

$$
\begin{aligned}
& \frac{z_{1}}{\varepsilon}=\cosh \left(\frac{S}{2}\right) \cos \left(\frac{\theta_{1}+\theta_{2}}{2}\right) \cos \left(\frac{\phi_{1}+\phi_{2}}{2}\right)+i \sinh \left(\frac{S}{2}\right) \cos \left(\frac{\theta_{1}-\theta_{2}}{2}\right) \sin \left(\frac{\phi_{1}+\phi_{2}}{2}\right), \\
& \frac{z_{2}}{\varepsilon}=-\cosh \left(\frac{S}{2}\right) \cos \left(\frac{\theta_{1}+\theta_{2}}{2}\right) \sin \left(\frac{\phi_{1}+\phi_{2}}{2}\right)+i \sinh \left(\frac{S}{2}\right) \cos \left(\frac{\theta_{1}-\theta_{2}}{2}\right) \cos \left(\frac{\phi_{1}+\phi_{2}}{2}\right), \\
& \frac{z_{3}}{\varepsilon}=-\cosh \left(\frac{S}{2}\right) \sin \left(\frac{\theta_{1}+\theta_{2}}{2}\right) \cos \left(\frac{\phi_{1}-\phi_{2}}{2}\right)+i \sinh \left(\frac{S}{2}\right) \sin \left(\frac{\theta_{1}-\theta_{2}}{2}\right) \sin \left(\frac{\phi_{1}-\phi_{2}}{2}\right), \\
& \frac{z_{4}}{\varepsilon}=-\cosh \left(\frac{S}{2}\right) \sin \left(\frac{\theta_{1}+\theta_{2}}{2}\right) \sin \left(\frac{\phi_{1}-\phi_{2}}{2}\right)-i \sinh \left(\frac{S}{2}\right) \sin \left(\frac{\theta_{1}-\theta_{2}}{2}\right) \cos \left(\frac{\phi_{1}-\phi_{2}}{2}\right),
\end{aligned}
$$

with $S=\tau+\mathrm{i} \psi$. The $\mathbb{Z}_{2}$ symmetry is then realized as $\psi \rightarrow \psi+2 \pi$. It is convenient to define,

$$
\begin{aligned}
& e_{1}=-\sin \theta_{1} \mathrm{~d} \phi_{1}, \\
& e_{2}=\mathrm{d} \theta_{1}, \\
& e_{3}=\cos \psi \sin \theta_{2} \mathrm{~d} \phi_{2}-\sin \psi \mathrm{d} \theta_{2}, \\
& e_{4}=\sin \psi \sin \theta_{2} \mathrm{~d} \phi_{2}+\cos \psi \mathrm{d} \theta_{2}, \\
& e_{5}=\mathrm{d} \psi+\cos \theta_{1} \mathrm{~d} \phi_{1}+\cos \theta_{2} \mathrm{~d} \phi_{2} .
\end{aligned}
$$

The metric for the deformed conifold is diagonal in the basis of 1-forms given by [58]

$$
\begin{aligned}
& g_{1}=\frac{1}{\sqrt{2}}\left(e_{1}-e_{3}\right), \\
& g_{2}=\frac{1}{\sqrt{2}}\left(e_{2}-e_{4}\right), \\
& g_{3}=\frac{1}{\sqrt{2}}\left(e_{1}+e_{3}\right), \\
& g_{4}=\frac{1}{\sqrt{2}}\left(e_{2}+e_{4}\right), \\
& g_{5}=e_{5} .
\end{aligned}
$$


In terms of the complex coordinates, there are relatively simple expressions available for the SO (4) invariant 1-forms

$$
\mathrm{d} \tau=\frac{1}{\varepsilon^{2} \sinh \tau}\left(z_{i} \mathrm{~d} \bar{z}_{i}+\bar{z}_{i} \mathrm{~d} z_{i}\right), \quad g_{5}=\frac{\mathrm{i}}{\varepsilon^{2} \sinh \tau}\left(z_{i} \mathrm{~d} \bar{z}_{i}-\bar{z}_{i} \mathrm{~d} z_{i}\right) .
$$

Similarly, for the SO (4)-invariant 2-forms

$$
\begin{aligned}
g_{1} \wedge g_{2} & =\frac{\mathrm{i}(1+\cosh \tau)}{2 \varepsilon^{4} \sinh ^{3} \tau} \epsilon_{i j k l}\left(2 z_{i} \bar{z}_{j} \mathrm{~d} z_{k} \wedge \mathrm{d} \bar{z}_{l}-z_{i} \bar{z}_{j} \mathrm{~d} z_{k} \wedge \mathrm{d} z_{l}-z_{i} \bar{z}_{j} \mathrm{~d} \bar{z}_{k} \wedge \mathrm{d} \bar{z}_{l}\right), \\
g_{3} \wedge g_{4} & =\frac{\mathrm{i} \tanh \left(\frac{\tau}{2}\right)}{2 \varepsilon^{4} \sinh ^{2} \tau} \epsilon_{i j k l}\left(2 z_{i} \bar{z}_{j} \mathrm{~d} z_{k} \wedge \mathrm{d} \bar{z}_{l}+z_{i} \bar{z}_{j} \mathrm{~d} z_{k} \wedge \mathrm{d} z_{l}+z_{i} \bar{z}_{j} \mathrm{~d} \bar{z}_{k} \wedge \mathrm{d} \bar{z}_{l}\right), \\
g_{1} \wedge g_{3}+g_{2} \wedge g_{4} & =\frac{1}{\varepsilon^{4} \sinh ^{2} \tau} \epsilon_{i j k l}\left(-z_{i} \bar{z}_{j} \mathrm{~d} z_{k} \wedge \mathrm{d} z_{l}+z_{i} \bar{z}_{j} \mathrm{~d} \bar{z}_{k} \wedge \mathrm{d} \bar{z}_{l}\right), \\
g_{2} \wedge g_{3}+g_{4} \wedge g_{1} & =-\frac{2 \mathrm{i} \cosh \tau}{\varepsilon^{4} \sinh ^{3} \tau}\left(\bar{z}_{j} \mathrm{~d} z_{j}\right) \wedge\left(z_{i} \mathrm{~d} \bar{z}_{i}\right)+\frac{2 \mathrm{i}}{\varepsilon^{2} \sinh \tau} \mathrm{d} z_{i} \wedge \mathrm{d} \bar{z}_{i} .
\end{aligned}
$$

The other 1-forms $g_{i}$ do not seem to be as easily expressed in terms of the holomorphic coordinates. However, one can show that

$$
\begin{aligned}
g_{1}^{2}+g_{2}^{2}=-\frac{1}{2 \varepsilon^{4} \sinh ^{2}(\tau / 2) \sinh ^{2} \tau} & {\left[\left(\bar{z}_{i} \mathrm{~d} z_{i}\right)^{2}+\left(z_{i} \mathrm{~d} \bar{z}_{i}\right)^{2}+2 \cosh \tau\left(\bar{z}_{i} \mathrm{~d} z_{i}\right)\left(z_{i} \mathrm{~d} \bar{z}_{i}\right)\right.} \\
& \left.+\varepsilon^{2} \sinh ^{2} \tau\left(\mathrm{d} z_{i} \mathrm{~d} z_{i}+\mathrm{d} \bar{z}_{i} \mathrm{~d} \bar{z}_{i}-2 \mathrm{~d} z_{i} \mathrm{~d} \bar{z}_{i}\right)\right] \\
g_{3}^{2}+g_{4}^{2}=\frac{1}{2 \varepsilon^{4} \cosh ^{2}(\tau / 2) \sinh ^{2} \tau}[ & \left(\bar{z}_{i} \mathrm{~d} z_{i}\right)^{2}+\left(z_{i} \mathrm{~d} \bar{z}_{i}\right)^{2}-2 \cosh \tau\left(\bar{z}_{i} \mathrm{~d} z_{i}\right)\left(z_{i} \mathrm{~d} \bar{z}_{i}\right) \\
+ & \left.\varepsilon^{2} \sinh ^{2} \tau\left(\mathrm{d} z_{i} \mathrm{~d} z_{i}+\mathrm{d} \bar{z}_{i} \mathrm{~d} \bar{z}_{i}+2 \mathrm{~d} z_{i} \mathrm{~d} \bar{z}_{i}\right)\right]
\end{aligned}
$$

Using these expressions and the metric and fluxes given in Section 2.1, the components of the 3-form flux having mixed holomorphic and anti-holomorphic indices (with respect to the complex structure of the unperturbed KS solution) are

$$
\begin{aligned}
& G_{3}^{(2,1)}=\frac{M \alpha^{\prime}}{2 \varepsilon^{6}}\left[2\left(a_{1}^{+}+a_{2}^{+}\right)\left(\bar{z}_{m} \mathrm{~d} z_{m}\right) \wedge\left(\epsilon_{i j k \ell} z_{i} \bar{z}_{j} \mathrm{~d} z_{k} \wedge \mathrm{d} \bar{z}_{\ell}\right)\right. \\
& \left.+\left(a_{1}^{-}-a_{2}^{-}-a_{3}^{+}\right)\left(z_{m} \mathrm{~d} \bar{z}_{m}\right) \wedge\left(\epsilon_{i j k \ell} z_{i} \bar{z}_{j} \mathrm{~d} z_{k} \wedge \mathrm{d} z_{\ell}\right)\right], \\
& G_{3}^{(1,2)}=\frac{M \alpha^{\prime}}{2 \varepsilon^{6}}\left[2\left(a_{1}^{-}+a_{2}^{-}\right)\left(z_{m} \mathrm{~d} \bar{z}_{m}\right) \wedge\left(\epsilon_{i j k \ell} z_{i} \bar{z}_{j} \mathrm{~d} z_{k} \wedge \mathrm{d} \bar{z}_{\ell}\right)\right. \\
& \left.+\left(a_{1}^{+}-a_{2}^{+}-a_{3}^{-}\right)\left(\bar{z}_{m} \mathrm{~d} z_{m}\right) \wedge\left(\epsilon_{i j k \ell} z_{i} \bar{z}_{j} \mathrm{~d} \bar{z}_{k} \wedge \mathrm{d} \bar{z}_{\ell}\right)\right],
\end{aligned}
$$

with

$$
\begin{aligned}
& a_{1}^{ \pm}(\tau)=\frac{\tanh \frac{\tau}{2}}{2 \sinh ^{3} \tau}\left( \pm(1-F)+g_{s} \mathrm{e}^{-\Phi} k^{\prime}\right), \\
& a_{2}^{ \pm}(\tau)=\frac{1+\cosh \tau}{2 \sinh ^{4} \tau}\left( \pm F+g_{s} \mathrm{e}^{-\Phi} f^{\prime}\right), \\
& a_{3}^{ \pm}(\tau)=\frac{1}{\sinh ^{3} \tau}\left( \pm F^{\prime}+g_{s} \mathrm{e}^{-\Phi} \frac{k-f}{2}\right) .
\end{aligned}
$$


The components with pure holomorphic and pure anti-holomorphic indices are

$$
\begin{aligned}
G_{3}^{(3,0)} & =\frac{M \alpha^{\prime}}{2 \varepsilon^{6}}\left[\left((1-F) \frac{\tanh \frac{\tau}{2}}{2 \sinh ^{3} \tau}-F \frac{1+\cosh \tau}{2 \sinh ^{4} \tau}-\frac{F^{\prime}}{\sinh ^{3} \tau}\right)\right. \\
& \left.+g_{s} \mathrm{e}^{-\Phi}\left(-f^{\prime} \frac{1+\cosh \tau}{2 \sinh ^{4} \tau}+k^{\prime} \frac{\tanh \frac{\tau}{2}}{2 \sinh ^{3} \tau}+\frac{k-f}{2 \sinh ^{3} \tau}\right)\right]\left(\bar{z}_{m} \mathrm{~d} z_{m}\right) \wedge\left(\epsilon_{i j k \ell} z_{i} \bar{z}_{j} \mathrm{~d} z_{k} \wedge \mathrm{d} z_{\ell}\right), \\
G_{3}^{(0,3)} & =\frac{M \alpha^{\prime}}{2 \varepsilon^{6}}\left[-\left((1-F) \frac{\tanh \frac{\tau}{2}}{2 \sinh ^{3} \tau}-F \frac{1+\cosh \tau}{2 \sinh ^{4} \tau}-\frac{F^{\prime}}{\sinh ^{3} \tau}\right)\right. \\
& \left.+g_{s} \mathrm{e}^{-\Phi}\left(-f^{\prime} \frac{1+\cosh \tau}{2 \sinh ^{4} \tau}+k^{\prime} \frac{\tanh \frac{\tau}{2}}{2 \sinh ^{3} \tau}+\frac{k-f}{2 \sinh ^{3} \tau}\right)\right]\left(z_{m} \mathrm{~d} \bar{z}_{m}\right) \wedge\left(\epsilon_{i j k \ell} z_{i} \bar{z}_{j} \mathrm{~d} \bar{z}_{k} \wedge \mathrm{d} \bar{z}_{\ell}\right) .
\end{aligned}
$$

For general functions $p, b, q$, and $s$, the metric will no longer be Hermitian with respect to the complex structure of the deformed conifold. In general, the unwarped $6 \mathrm{D}$ metric takes the form

$$
\begin{aligned}
\left(\varepsilon^{4} \sinh ^{2} \tau\right) \mathrm{d} \tilde{s}_{6}^{2} & =\left\{p(\tau)-b(\tau)+\frac{1}{2}\left[\frac{q(\tau)}{\cosh ^{2}(\tau / 2)}-\frac{s(\tau)}{\sinh ^{2}(\tau / 2)}\right]\right\}\left(\left(\bar{z}_{i} \mathrm{~d} z_{i}\right)^{2}+\left(z_{i} \mathrm{~d} \bar{z}_{i}\right)^{2}\right) \\
& +\frac{1}{2} \varepsilon^{2} \sinh ^{2} \tau\left[\frac{q(\tau)}{\cosh ^{2}(\tau / 2)}-\frac{s(\tau)}{\sinh ^{2}(\tau / 2)}\right]\left(\mathrm{d} z_{i} \mathrm{~d} z_{i}+\mathrm{d} \bar{z}_{i} \mathrm{~d} \bar{z}_{i}\right) \\
& +2\left\{p(\tau)+b(\tau)-\frac{1}{2} \cosh (\tau)\left[\frac{q(\tau)}{\cosh ^{2}(\tau / 2)}+\frac{s(\tau)}{\sinh ^{2}(\tau / 2)}\right]\right\}\left(\bar{z}_{i} \mathrm{~d} z_{i}\right)\left(z_{i} \mathrm{~d} \bar{z}_{i}\right) \\
& +\varepsilon^{2} \sinh ^{2} \tau\left[\frac{q(\tau)}{\cosh ^{2}(\tau / 2)}+\frac{s(\tau)}{\sinh ^{2}(\tau / 2)}\right] \mathrm{d} z_{i} \mathrm{~d} \bar{z}_{i} .
\end{aligned}
$$

The holomorphic 3-form for the deformed conifold is

$$
\begin{gathered}
\Omega=\frac{\varepsilon^{2}}{16 \sqrt{3}}\left[-\sinh \tau\left(g_{1} \wedge g_{3}+g_{2} \wedge g_{4}\right)+\mathrm{i} \cosh \tau\left(g_{1} \wedge g_{2}-g_{3} \wedge g_{4}\right)\right. \\
\left.-\mathrm{i}\left(g_{1} \wedge g_{2}+g_{3} \wedge g_{4}\right)\right] \wedge\left(\mathrm{d} \tau+\mathrm{i} g_{5}\right) \\
=\frac{1}{4 \sqrt{3} \varepsilon^{4} \sinh ^{2} \tau}\left(\epsilon_{i j k l} z_{i} \bar{z}_{j} \mathrm{~d} z_{k} \wedge \mathrm{d} z_{l}\right) \wedge\left(\bar{z}_{m} \mathrm{~d} z_{m}\right) .
\end{gathered}
$$

It also convenient to introduce another set of holomorphic 1-forms

$$
\begin{aligned}
\mathrm{d} Z_{1} & =\mathrm{d} \tau+\mathrm{i} g_{5}, \\
\mathrm{~d} Z_{2} & =g_{1}-\mathrm{i} \operatorname{coth} \frac{\tau}{2} g_{4}, \\
\mathrm{~d} Z_{3} & =g_{3}-\mathrm{i} \tanh \frac{\tau}{2} g_{2} .
\end{aligned}
$$


In these coordinates the metric (2.16b) is written

$$
\begin{aligned}
\mathrm{d} \tilde{s}_{6}^{2} & =\frac{1}{2}(p(\tau)+b(\tau)) \mathrm{d} Z_{1} \mathrm{~d} \bar{Z}_{1}+\frac{1}{2}\left(s(\tau)+q(\tau) \tanh ^{2} \frac{\tau}{2}\right) \mathrm{d} Z_{2} \mathrm{~d} \bar{Z}_{2} \\
+ & \frac{1}{2}\left(s(\tau) \operatorname{coth}^{2} \frac{\tau}{2}+q(\tau)\right) \mathrm{d} Z_{3} \mathrm{~d} \bar{Z}_{3} \\
& +\frac{1}{4}(p(\tau)-b(\tau))\left(\mathrm{d} Z_{1} \mathrm{~d} Z_{1}+\mathrm{d} \bar{Z}_{1} \mathrm{~d} \bar{Z}_{1}\right)+\frac{1}{4}\left(s(\tau)-q(\tau) \tanh ^{2} \frac{\tau}{2}\right)\left(\mathrm{d} Z_{2} \mathrm{~d} Z_{2}+\mathrm{d} \bar{Z}_{2} \mathrm{~d} \bar{Z}_{2}\right) \\
& +\frac{1}{4}\left(-s(\tau) \operatorname{coth}^{2} \frac{\tau}{2}+q(\tau)\right)\left(\mathrm{d} Z_{3} \mathrm{~d} Z_{3}+\mathrm{d} \bar{Z}_{3} \mathrm{~d} \bar{Z}_{3}\right)
\end{aligned}
$$

and the holomorphic 3-form of the deformed conifold takes the simple form

$$
\Omega=-\frac{\varepsilon^{2}}{16 \sqrt{3}} \sinh \tau \mathrm{d} Z_{1} \wedge \mathrm{d} Z_{2} \wedge \mathrm{d} Z_{3} .
$$

The components of $G_{3}$ with mixed holomorphic and anti-holomorphic indices can then be written

$$
\begin{aligned}
& G_{3}^{(2,1)}=-\frac{M \alpha^{\prime} \sinh ^{3} \tau}{8}\left\{\left(a_{1}^{+}+a_{2}^{+}\right)\left(\mathrm{d} Z_{1} \wedge \mathrm{d} Z_{2} \wedge \mathrm{d} \bar{Z}_{3}-\mathrm{d} Z_{1} \wedge \mathrm{d} \bar{Z}_{2} \wedge \mathrm{d} Z_{3}\right)\right. \\
& \left.+\left(a_{1}^{-}-a_{2}^{-}-a_{3}^{+}\right) \mathrm{d} \bar{Z}_{1} \wedge \mathrm{d} Z_{2} \wedge \mathrm{d} Z_{3}\right\}, \\
& G_{3}^{(1,2)}=\frac{M \alpha^{\prime} \sinh ^{3} \tau}{8}\left\{\left(a_{1}^{-}+a_{2}^{-}\right)\left(\mathrm{d} \bar{Z}_{1} \wedge \mathrm{d} \bar{Z}_{2} \wedge \mathrm{d} Z_{3}-\mathrm{d} \bar{Z}_{1} \wedge \mathrm{d} Z_{2} \wedge \mathrm{d} \bar{Z}_{3}\right)\right. \\
& \left.+\left(a_{1}^{+}-a_{2}^{+}-a_{3}^{-}\right) \mathrm{d} Z_{1} \wedge \mathrm{d} \bar{Z}_{2} \wedge \mathrm{d} \bar{Z}_{3}\right\}
\end{aligned}
$$

while the components with pure holomorphic or pure anti-holomorphic indices are

$$
\begin{aligned}
G_{3}^{(3,0)}=\frac{M \alpha^{\prime}}{16}\{ & -(1-F) \tanh \frac{\tau}{2}+F \operatorname{coth} \frac{\tau}{2}+2 F^{\prime} \\
& \left.+g_{s} \mathrm{e}^{-\Phi}\left(f^{\prime} \operatorname{coth} \frac{\tau}{2}-k^{\prime} \tanh \frac{\tau}{2}-(f-k)\right)\right\} \mathrm{d} Z_{1} \wedge \mathrm{d} Z_{2} \wedge \mathrm{d} Z_{3}, \\
G_{3}^{(0,3)}=\frac{M \alpha^{\prime}}{16}\{ & -(1-F) \tanh \frac{\tau}{2}+F \operatorname{coth} \frac{\tau}{2}+2 F^{\prime} \\
& \left.-g_{s} \mathrm{e}^{-\Phi}\left(f^{\prime} \operatorname{coth} \frac{\tau}{2}-k^{\prime} \tanh \frac{\tau}{2}-(f-k)\right)\right\} \mathrm{d} \bar{Z}_{1} \wedge \mathrm{d} \bar{Z}_{2} \wedge \mathrm{d} \bar{Z}_{3} .
\end{aligned}
$$




\section{Pullbacks of bulk fields}

We consider a stack of D7-branes satisfying the Kuperstein embedding condition 34]

$$
z_{4}=\mu
$$

where $z_{i}$ are holomorphic coordinates satisfying (B.1). For the purpose of computing pullbacks onto the world-volumes, it is useful to adopt coordinates in which the bulk geometry is seen as a foliation of Kuperstein divisors. Such coordinates $(\rho, \chi, \bar{\chi}, \phi, \theta, \xi)$ were given in [25]

$$
\begin{aligned}
& z_{1}=\mathrm{i} \eta(\chi)\left[\cos \phi \cosh \left(\frac{\rho+\mathrm{i} \xi}{2}\right) \cos \theta-\mathrm{i} \sin \phi \sinh \left(\frac{\rho+\mathrm{i} \xi}{2}\right)\right] \\
& z_{2}=\mathrm{i} \eta(\chi)\left[\sin \phi \cosh \left(\frac{\rho+\mathrm{i} \xi}{2}\right) \cos \theta-\mathrm{i} \cos \phi \sinh \left(\frac{\rho+\mathrm{i} \xi}{2}\right)\right] \\
& z_{3}=\mathrm{i} \eta(\chi) \cosh \left(\frac{\rho+\mathrm{i} \xi}{2}\right) \sin \theta \\
& z_{4}=\mu+\chi
\end{aligned}
$$

where the $z_{i}$ still satisfy (B.1). $\eta$ and $\rho$ are defined by

$$
\eta(\chi)=\sqrt{(\mu+\chi)^{2}-\varepsilon^{2}}
$$

and $\rho$ is given by

$$
\varepsilon^{2} \cosh \tau=|\eta|^{2} \cosh \rho+|\mu+\chi|^{2} .
$$

A Kuperstein embedding is then specified by the simple condition $\chi=0$. One can find the unwarped metric on the 4-cycle wrapped by the D7-branes by substituting the coordinates (C.2) into the metric (B.12). The result is

$$
\mathrm{d} \tilde{s}_{4}^{2}=v(\tau) \mathrm{d} \rho^{2}+w(\tau) h_{3}^{2}+\frac{\eta^{2} s(\tau)(\cosh \rho+1)}{2 \varepsilon^{2}(\cosh \tau-1)} h_{1}^{2}+\frac{\eta^{2} q(\tau)(\cosh \rho-1)}{2 \varepsilon^{2}(\cosh \tau+1)} h_{2}^{2},
$$

with

$$
\begin{array}{r}
v(\tau)=\frac{\eta^{2}}{2 \varepsilon^{2}}\left\{\frac{2 \eta^{2} p(\tau) \sinh ^{2} \rho}{\sinh ^{2} \tau}+q(\tau)\left[\frac{\varepsilon^{2}(\cosh \rho+1)}{\cosh \tau+1}-\frac{\eta^{2} \sinh ^{2} \rho}{(\cosh \tau+1)^{2}}\right]\right. \\
\left.+s(\tau)\left[\frac{\varepsilon^{2}(\cosh \rho-1)}{\cosh \tau-1}-\frac{\eta^{2} \sinh ^{2} \rho}{(\cosh \tau-1)^{2}}\right]\right\}, \\
\begin{aligned}
w(\tau)=\frac{\eta^{2}}{2 \varepsilon^{2}}\left\{\frac{2 \eta^{2} b(\tau) \sinh ^{2} \rho}{\sinh ^{2} \tau}+q(\tau)\left[\frac{\varepsilon^{2}(\cosh \rho-1)}{\cosh \tau+1}-\frac{\eta^{2} \sinh ^{2} \rho}{\sinh ^{2} \tau}\right]\right. \\
\left.+s(\tau)\left[\frac{\varepsilon^{2}(\cosh \rho+1)}{\cosh \tau-1}-\frac{\eta^{2} \sinh ^{2} \rho}{\sinh ^{2} \tau}\right]\right\}
\end{aligned}
\end{array}
$$


where as in 25], the 1 -forms $h_{i}$ are

$$
\begin{aligned}
& h_{1}=2\left(\cos \frac{\gamma}{2} \mathrm{~d} \theta-\sin \frac{\gamma}{2} \sin \theta \mathrm{d} \phi\right), \\
& h_{2}=2\left(\sin \frac{\gamma}{2} \mathrm{~d} \theta+\cos \frac{\gamma}{2} \sin \theta \mathrm{d} \phi\right), \\
& h_{3}=\mathrm{d} \gamma-2 \cos \theta \mathrm{d} \phi .
\end{aligned}
$$

For the KS geometry, this reduces to the metric given in 25],

$$
\mathrm{d} \tilde{s}_{4}^{2}=\frac{K(\tau) \eta^{2}}{2 \varepsilon^{2 / 3}}\left[K_{2}(\rho)\left(\mathrm{d} \rho^{2}+h_{3}^{2}\right)+\cosh ^{2} \frac{\rho}{2} h_{1}^{2}+\sinh ^{2} \frac{\rho}{2} h_{2}^{2}\right]
$$

The pullback of $B_{(2)}$ can also be written in these coordinates as

$$
\begin{array}{r}
b_{2}=-\frac{g_{s} M \alpha^{\prime}}{2 \varepsilon^{4}} \eta^{3} \mu[ \\
k(\tau) \operatorname{csch} \frac{\rho}{2} \operatorname{csch}^{3} \tau \sinh ^{2} \rho \sinh ^{2} \frac{\tau}{2} \mathrm{~d} \rho \wedge h_{2} \\
\left.+\frac{1}{2} f(\tau) \cosh ^{3} \frac{\rho}{2} \operatorname{csch}^{3} \frac{\tau}{2} \operatorname{sech} \frac{\tau}{2} h_{1} \wedge h_{3}\right] .
\end{array}
$$

\section{References}

[1] S. P. Martin, A supersymmetry primer, hep-ph/9709356.

[2] D. Chung, L. Everett, G. Kane, S. King, and J. D. Lykken, The soft supersymmetry-breaking Lagrangian: Theory and applications, Phys. Rept. 407 (2005) 1-203, hep-ph/0312378].

[3] M. Dine, W. Fischler, and M. Srednicki, Supersymmetric technicolor, Nucl. Phys. B189 (1981) $575-593$.

[4] S. Dimopoulos and S. Raby, Supercolor, Nucl. Phys. B192 (1981) 353.

[5] M. Dine and W. Fischler, A phenomenological model of particle physics based on supersymmetry, Phys. Lett. B110 (1982) 227.

[6] C. R. Nappi and B. A. Ovrut, Supersymmetric extension of the $\mathrm{SU}(3) \times \mathrm{SU}(2) \times \mathrm{U}(1)$ model, Phys. Lett. B113 (1982) 175.

[7] L. Alvarez-Gaume, M. Claudson, and M. B. Wise, Low-Energy Supersymmetry, Nucl. Phys. B207 (1982) 96.

[8] S. Dimopoulos and S. Raby, Geometric hierarchy, Nucl. Phys. B219 (1983) 479.

[9] M. Dine and A. E. Nelson, Dynamical supersymmetry breaking at low-energies, Phys. Rev. D48 (1993) 1277-1287, hep-ph/9303230.

[10] M. Dine, A. E. Nelson, and Y. Shirman, Low-energy dynamical supersymmetry breaking simplified, Phys. Rev. D51 (1995) 1362-1370, hep-ph/9408384.

[11] M. Dine, A. E. Nelson, Y. Nir, and Y. Shirman, New tools for low-energy dynamical supersymmetry breaking, Phys. Rev. D53 (1996) 2658-2669, hep-ph/9507378. 
[12] G. F. Giudice and R. Rattazzi, Theories with gauge-mediated supersymmetry breaking, Phys. Rept. 322 (1999) 419-499, hep-ph/9801271.

[13] P. Meade, N. Seiberg, and D. Shih, General gauge mediation, Prog. Theor. Phys. Suppl. 177 (2009) 143-158, arXiv:0801.3278.

[14] Y. Shadmi and Y. Shirman, Dynamical supersymmetry breaking, Rev. Mod. Phys. 72 (2000) 25-64, hep-th/9907225.

[15] I. Affleck, M. Dine, and N. Seiberg, Dynamical supersymmetry breaking in four-dimensions and its phenomenological implications, Nucl. Phys. B256 (1985) 557.

[16] E. Poppitz and S. P. Trivedi, New models of gauge and gravity mediated supersymmetry breaking, Phys. Rev. D55 (1997) 5508-5519, hep-ph/9609529.

[17] N. Arkani-Hamed, J. March-Russell, and H. Murayama, Building models of gauge-mediated supersymmetry breaking without a messenger sector, Nucl. Phys. B509 (1998) 3-32, hep-ph/9701286.

[18] H. Murayama, A model of direct gauge mediation, Phys. Rev. Lett. 79 (1997) 18-21, hep-ph/9705271.

[19] N. Seiberg, T. Volansky, and B. Wecht, Semi-direct gauge mediation, JHEP 11 (2008) 004, arXiv:0809.4437.

[20] N. Seiberg, Electric - magnetic duality in supersymmetric non-abelian gauge theories, Nucl. Phys. B435 (1995) 129-146, hep-th/9411149.

[21] J. M. Maldacena, The large $N$ limit of superconformal field theories and supergravity, Adv. Theor. Math. Phys. 2 (1998) 231-252, hep-th/9711200.

[22] S. S. Gubser, I. R. Klebanov, and A. M. Polyakov, Gauge theory correlators from non-critical string theory, Phys. Lett. B428 (1998) 105-114, hep-th/9802109.

[23] E. Witten, Anti-de Sitter space and holography, Adv. Theor. Math. Phys. 2 (1998) 253-291, hep-th/9802150.

[24] S. Kachru, D. Simic, and S. P. Trivedi, Stable non-supersymmetric throats in string theory, arXiv:0905.2970.

[25] F. Benini, A. Dymarsky, S. Franco, S. Kachru, D. Simic, and H. Verlinde, Holographic gauge mediation, arXiv:0903.0619.

[26] P. McGuirk, G. Shiu, and Y. Sumitomo, Non-supersymmetric infrared perturbations to the warped deformed conifold, arXiv:0910.4581.

[27] O. DeWolfe, S. Kachru, and M. Mulligan, A gravity dual of metastable dynamical supersymmetry breaking, Phys. Rev. D77 (2008) 065011, arXiv:0801.1520.

[28] K. I. Izawa and Y. Nakai, Strongly coupled semi-direct mediation of supersymmetry breaking, arXiv:0812.4089.

[29] I. R. Klebanov and A. A. Tseytlin, Gravity duals of supersymmetric $\mathrm{SU}(N) \times \mathrm{SU}(N+M)$ gauge theories, Nucl. Phys. B578 (2000) 123-138, hep-th/0002159.

[30] I. R. Klebanov and M. J. Strassler, Supergravity and a confining gauge theory: Duality cascades and $\chi S B$-resolution of naked singularities, JHEP 08 (2000) 052, hep-th/0007191. 
[31] S. Kachru, J. Pearson, and H. L. Verlinde, Brane/flux annihilation and the string dual of a nonsupersymmetric field theory, JHEP 06 (2002) 021, hep-th/0112197.

[32] A. Karch and E. Katz, Adding flavor to AdS/CFT, JHEP 06 (2002) 043, hep-th/0205236.

[33] P. Ouyang, Holomorphic D\%-branes and flavored $\mathcal{N}=1$ gauge theories, Nucl. Phys. B699 (2004) 207-225, hep-th/0311084.

[34] S. Kuperstein, Meson spectroscopy from holomorphic probes on the warped deformed conifold, JHEP 03 (2005) 014, hep-th/0411097.

[35] H.-Y. Chen, P. Ouyang, and G. Shiu, On supersymmetric D\%-branes in the warped deformed conifold, arXiv:0807.2428.

[36] C. P. Herzog, I. R. Klebanov, and P. Ouyang, Remarks on the warped deformed conifold, hep-th/0108101.

[37] I. R. Klebanov, P. Ouyang, and E. Witten, A gravity dual of the chiral anomaly, Phys. Rev. D65 (2002) 105007, hep-th/0202056.

[38] G. 't Hooft, A planar diagram theory for strong interactions, Nucl. Phys. B72 (1974) 461.

[39] G. 't Hooft, A two-dimensional model for mesons, Nucl. Phys. B75 (1974) 461.

[40] E. Witten, Baryons in the 1/N expansion, Nucl. Phys. B160 (1979) 57.

[41] D. E. Kaplan, G. D. Kribs, and M. Schmaltz, Supersymmetry breaking through transparent extra dimensions, Phys. Rev. D62 (2000) 035010, hep-ph/9911293.

[42] Z. Chacko, M. A. Luty, A. E. Nelson, and E. Ponton, Gaugino mediated supersymmetry breaking, JHEP 01 (2000) 003, hep-ph/9911323.

[43] P. G. Camara, L. E. Ibanez, and A. M. Uranga, Flux-induced SUSY-breaking soft terms on D7-D3 brane systems, Nucl. Phys. B708 (2005) 268-316, hep-th/0408036.

[44] D. Lust, F. Marchesano, L. Martucci, and D. Tsimpis, Generalized non-supersymmetric flux vacua, JHEP 11 (2008) 021, arXiv:0807.4540.

[45] F. Benini, F. Canoura, S. Cremonesi, C. Nunez, and A. V. Ramallo, Backreacting flavors in the Klebanov-Strassler background, JHEP 09 (2007) 109, arXiv:0706.1238.

[46] F. Benini, A chiral cascade via backreacting D7-branes with flux, JHEP 10 (2008) 051, arXiv:0710.0374.

[47] L. Martucci, J. Rosseel, D. Van den Bleeken, and A. Van Proeyen, Dirac actions for D-branes on backgrounds with fluxes, Class. Quant. Grav. 22 (2005) 2745-2764, hep-th/0504041.

[48] D. Marolf, L. Martucci, and P. J. Silva, Fermions, T-duality and effective actions for D-branes in bosonic backgrounds, JHEP 04 (2003) 051, hep-th/0303209.

[49] D. Marolf, L. Martucci, and P. J. Silva, Actions and fermionic symmetries for D-branes in bosonic backgrounds, JHEP 07 (2003) 019, hep-th/0306066.

[50] F. Marchesano, P. McGuirk, and G. Shiu, Open string wavefunctions in warped compactifications, JHEP 04 (2009) 095, arXiv:0812.2247.

[51] P. G. Camara, L. E. Ibanez, and A. M. Uranga, Flux-induced SUSY-breaking soft terms, Nucl. Phys. B689 (2004) 195-242, hep-th/0311241. 
[52] M. Marino, R. Minasian, G. W. Moore, and A. Strominger, Nonlinear instantons from supersymmetric p-branes, JHEP 01 (2000) 005, hep-th/9911206.

[53] J. Gomis, F. Marchesano, and D. Mateos, An open string landscape, JHEP 11 (2005) 021, hep-th/0506179.

[54] M. Ibe, K. I. Izawa, and Y. Nakai, Studying gaugino mass in semi-direct gauge mediation, Phys. Rev. D80 (2009) 035002, arXiv:0907.2970.

[55] J. Polchinski and M. J. Strassler, The string dual of a confining four-dimensional gauge theory, hep-th/0003136.

[56] R. C. Myers, Dielectric-branes, JHEP 12 (1999) 022, hep-th/9910053.

[57] P. Candelas and X. C. de la Ossa, Comments on conifolds, Nucl. Phys. B342 (1990) 246-268.

[58] R. Minasian and D. Tsimpis, On the geometry of non-trivially embedded branes, Nucl. Phys. B572 (2000) 499-513, hep-th/9911042. 